C-A/AP/\#167

September 2004

\title{
Matching the Cold AGS Snake to the AGS Lattice
}

\author{
A. Luccio, T. Roser
}

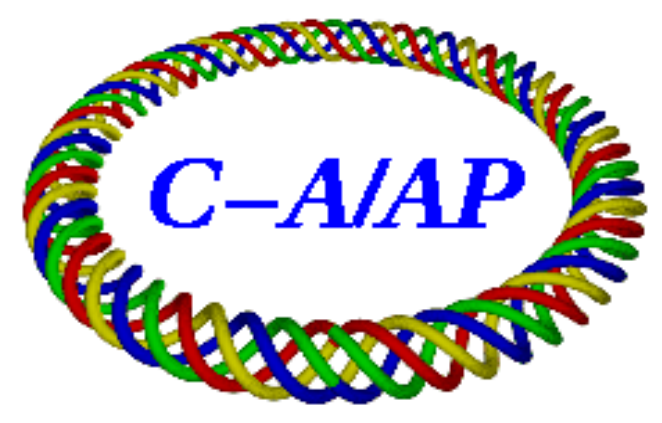

Collider-Accelerator Department

Brookhaven National Laboratory

Upton, NY 11973 


\title{
Matching the Cold AGS Snake to the AGS lattice
}

\author{
A.U. Luccio, T. Roser
}

September 14, 2004

\section{Introduction}

Ernest Courant proposed and worked out a solution of matching of the cold snake to the AGS lattice using four quadrupoles [1]. The present report is a remake of his work, using a realistic "Bleser" lattice for the AGS and a set of transfer matrices for the snake at different energies. The snake model was described in a previous CAP note [2]. Our purpose is to specify the value of the excitation of the four added quadrupoles and of the AGS tune quadrupoles in preparation of the commissioning of the snake. Two of the four quadrupoles will be obtained by additional currents in two of the already existing tune quadrupoles; two extra quadrupoles will be fabricated.

The four matching quads will be located located in A17, A19, B1 and B3 in the AGS, as shown in Fig. 1. QCA17 and QCB3 will be obtained by extra windings on the tune quads, QHFH and QHFV at the same locations. Space is very tight in the AGS, so the total length of the new fabricated quadrupoles at A19 and B1 is limited to $0.35 \mathrm{~m}$, see Figs. 2,3,4,5. Note that at the present time the gap at A19 is occupied by an octupole. As a reference, the length of the existing tune quads is 0.39088 $\mathrm{m}(15.4 \mathrm{in})$.

\section{MAD procedure}

1. Match bare insertion. (MAD commands in Italics.)

- Use $M A D 8$

- Use Bleser AGS lattice (e.g. /home/luccio/MAD/AGS/T-Snake/lattice/ags.lat).

- Start with bare machine (no snake), called AGS (use, period=A20DUM, super=12). This is the insert line with no snake.

- With cell find the tune quad currents XQH and XQV that produce a given tune, say $Q_{x}=8.78$, $Q_{y}=8.90$ in the vhole machine, or $Q_{x} / 12, Q_{y} / 12$ in the period.

- Save twiss functions (savebeta) at both ends of line.

2. Match insertion with snake

- Insert snake represented by a matrix in MAD format. Matrices calculated with Snig at different beam energies are stored in /home/luccio/MAD/AGS/T-Snake/TMaps/

- See first what happens doing nothing.

- Do insertion match using the four matching quads. (use period=A20SNK0 and match line=A20DUM).

I.e. match the line with snake to the line previouslty tuned with no snake

- vary KCA17, KCA19, KCB1, KCB3.

- Constraints at the end of line are the ones previously saved - we want the rest of the lattice to look like before the snake was inserted. 


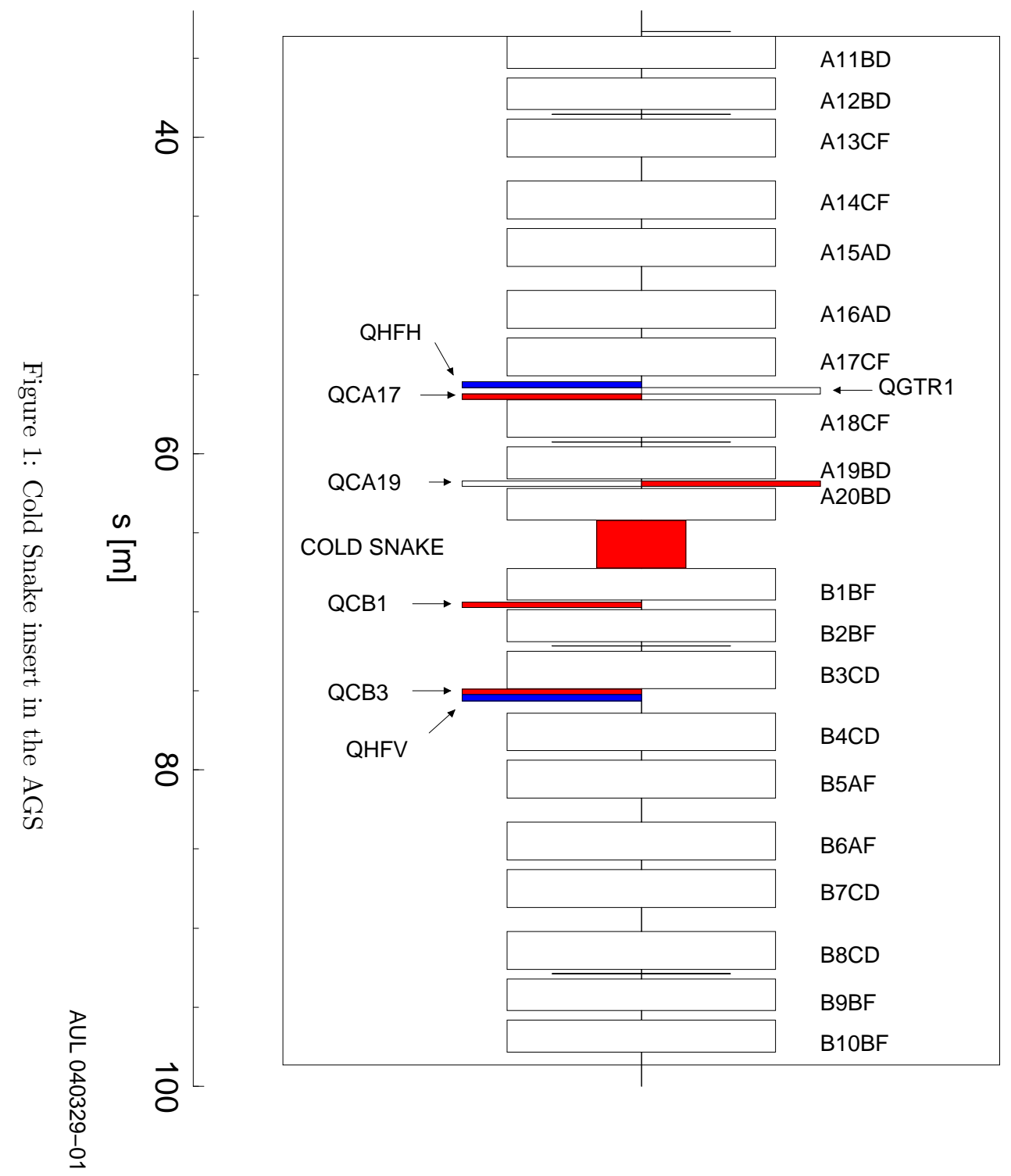




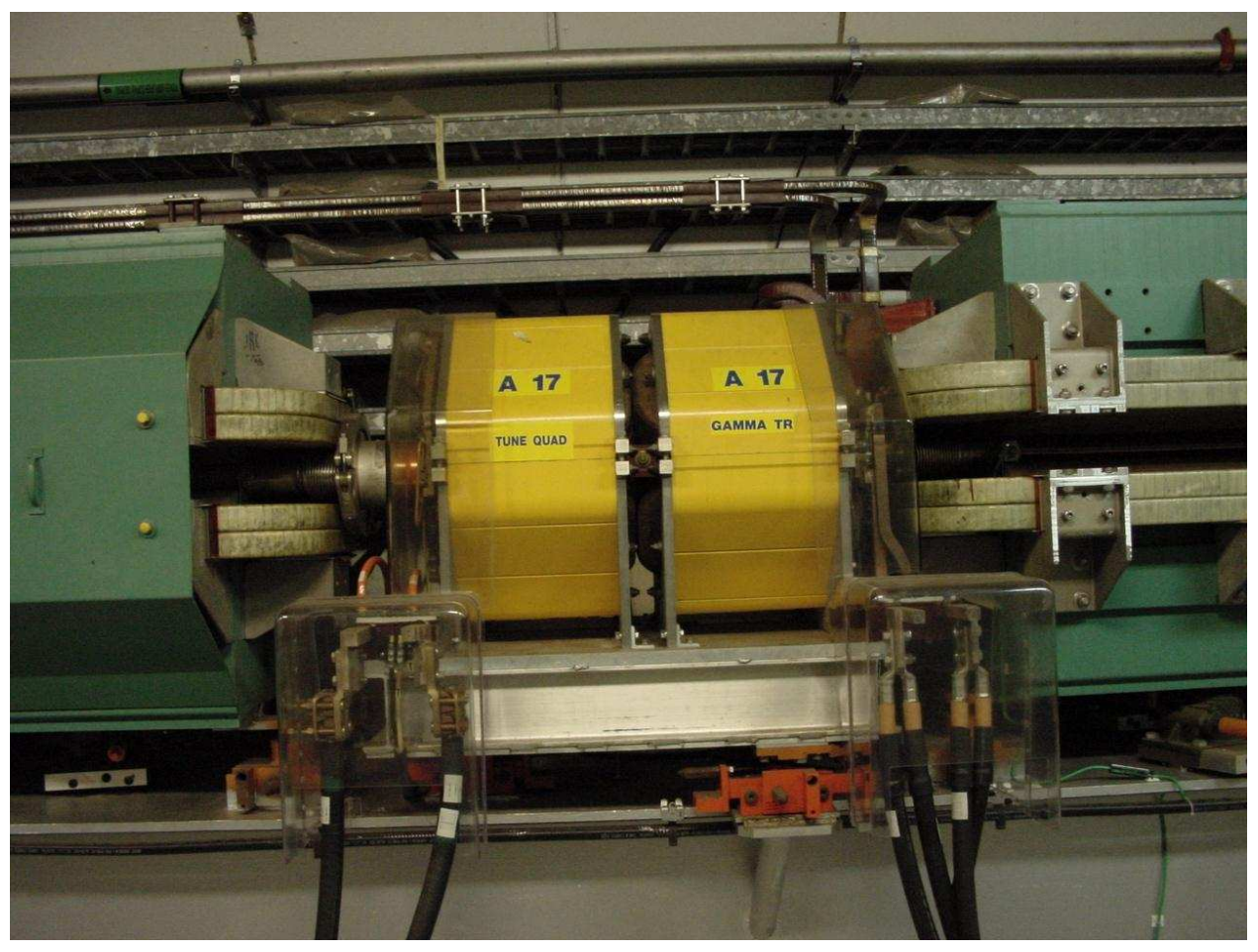

Figure 2: AGS: gap at A17

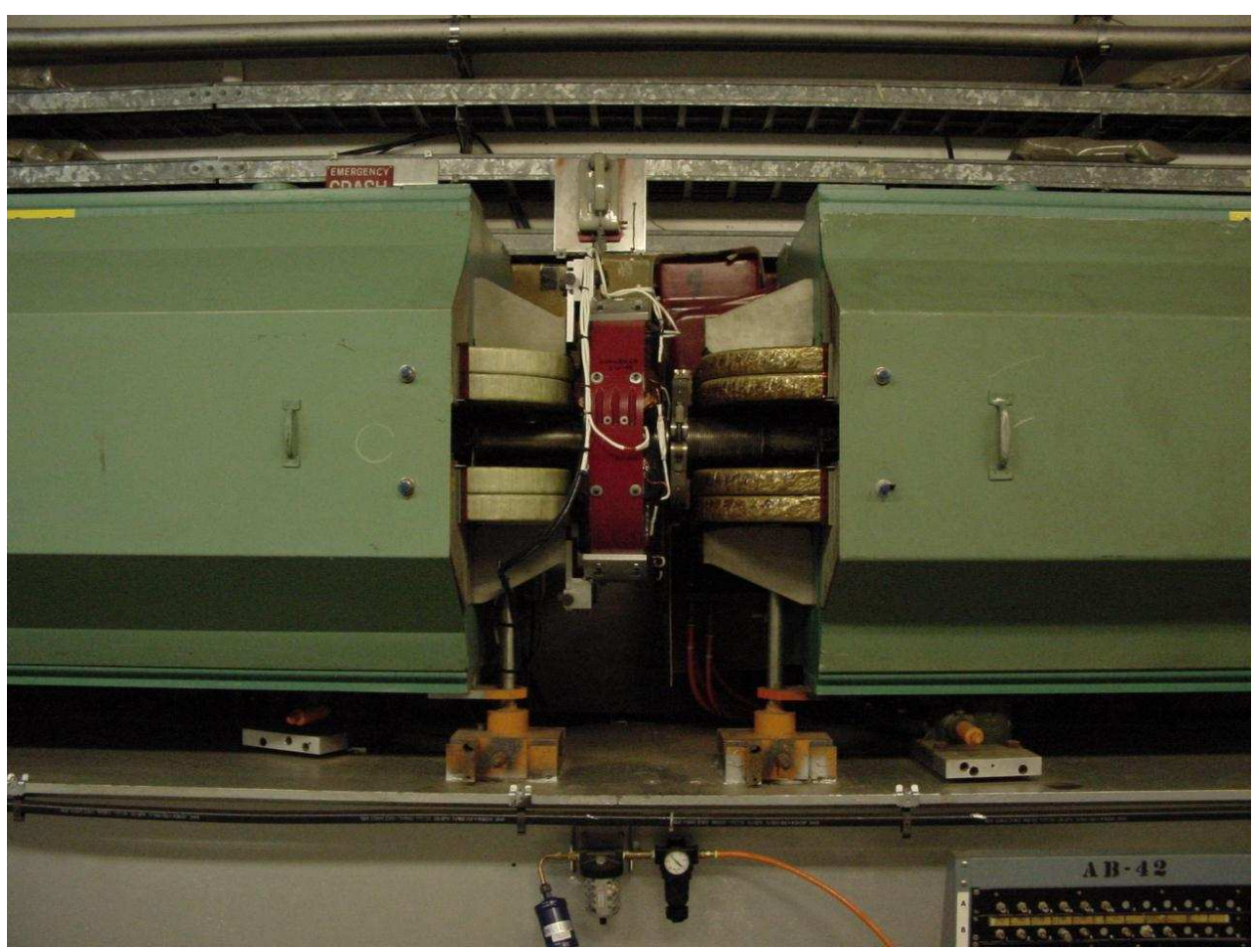

Figure 3: AGS: gap at A19 


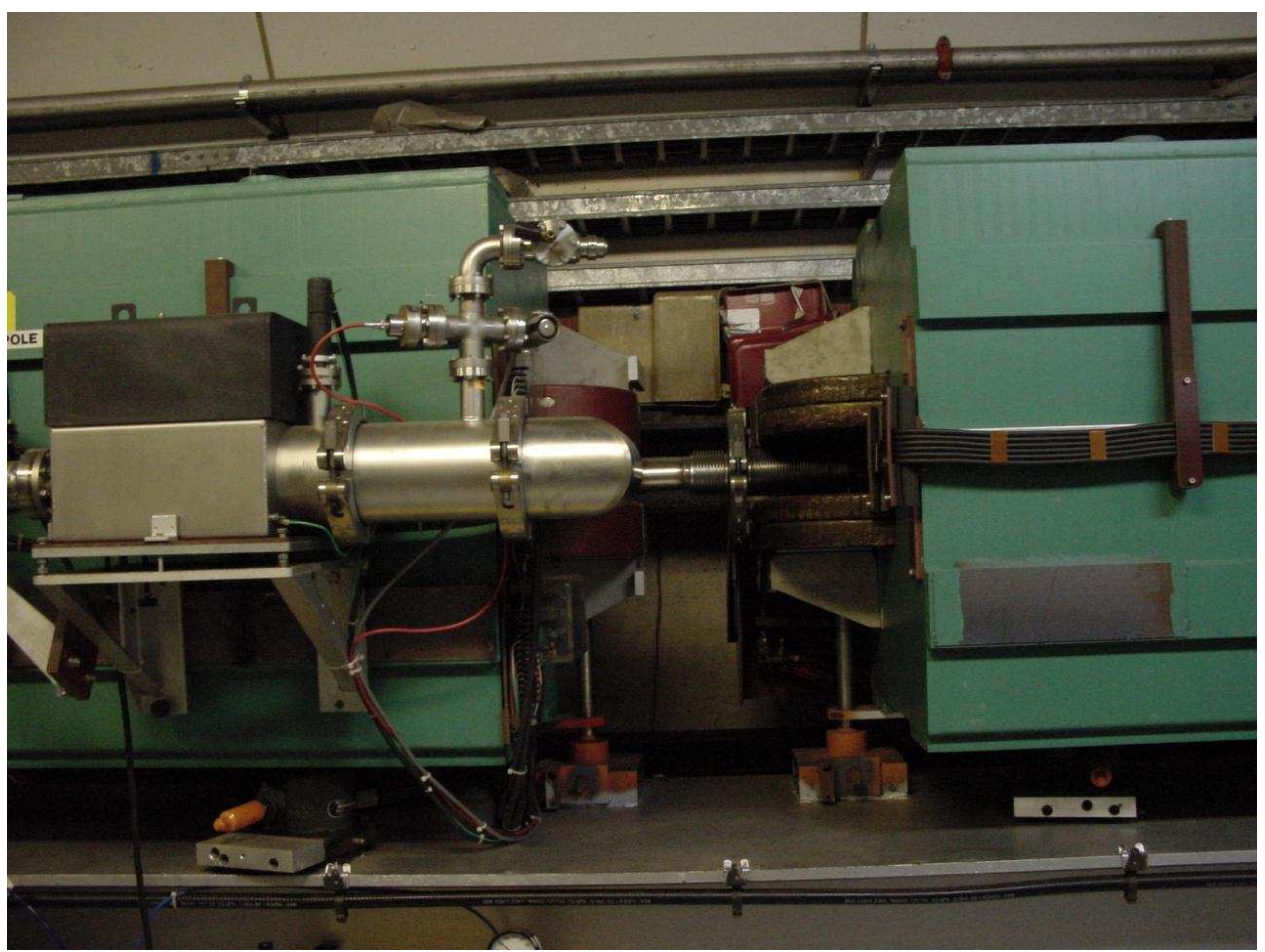

Figure 4: AGS: gap at B1

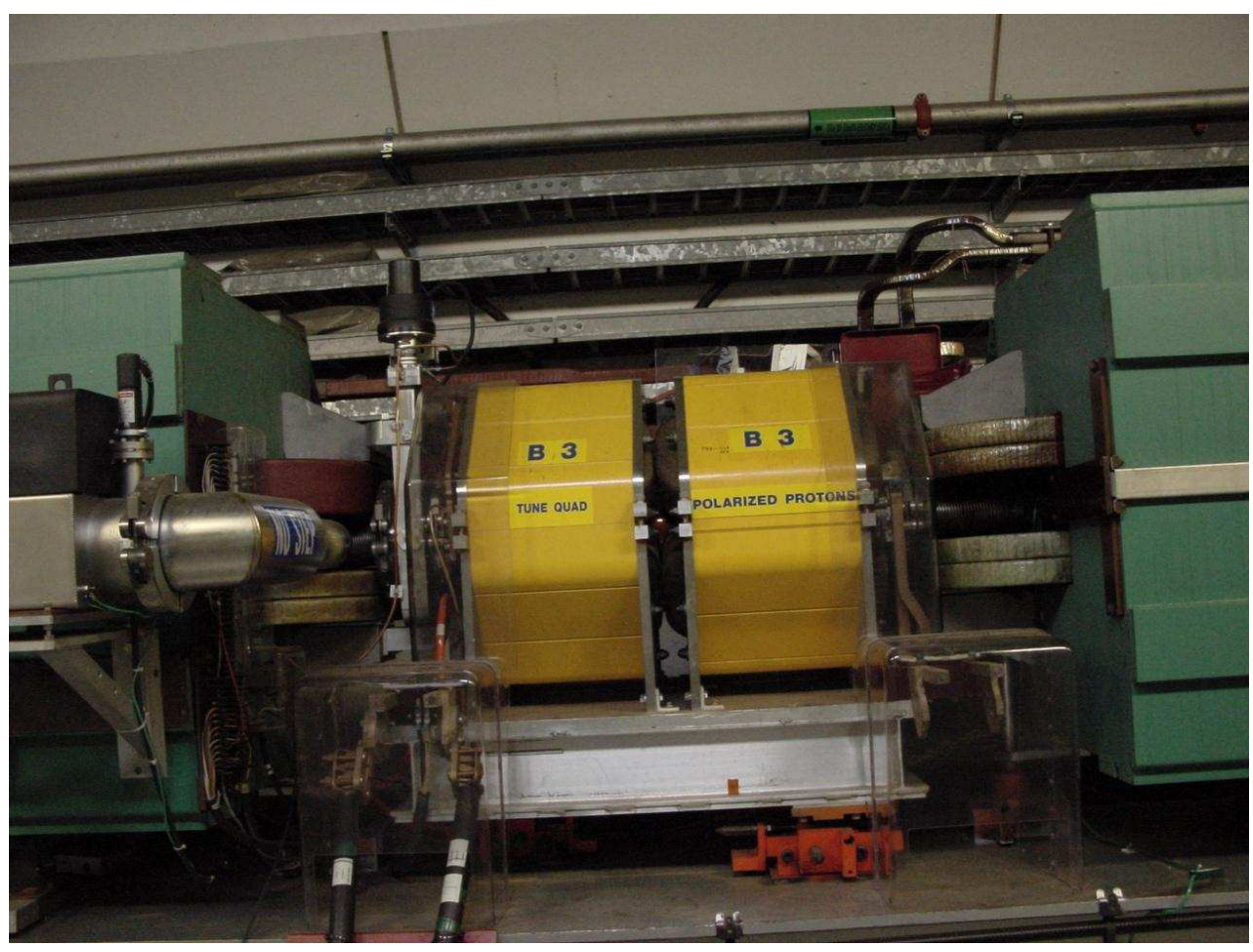

Figure 5: AGS: gap at B3 


\section{Retune}

- use, AGS-SA, i.e. the whole machine containing the snake.

- Varying tune quads, with constraints for the maximum beta values outside the snake insertion. Betas may go crazy inside, but they must remain limited in the rest of the ring.

\section{Snake Transfer Maps}

$4 \times 4$ transverse transfer linear maps have been described in [2]. The snake, which is represented in the $M A D$ model as a $3 \mathrm{~m}$ long matrix, becomes more and more transparent with increasing beam energy: see plots of the individual matrix elements as a function of energy in Fig. 6 .

Among the matrix elements, important are the $R(2,1)$, giving the horizontal focusing of the snake, and $R(4,3)$ that gives the vertical focusing, respectively. They are shown again in Fig. 7 . It is important to realize that these focusing elements scale with energy almost exactly as the reciprocal of the beam rigidity $B \rho$ squared, as expected, because the orbit amplitude in the helical snake grows linearly with the energy, but the transverse magnetic field on the orbit scales with the square of the orbit displacement [2].

\section{At injection: $\gamma=2.5$}

At injection, at the lowest end of energy, matching the cold snake to the AGS lattice using the four matching quadrupoles proves praticularly challenging, since both snake focusing and coupling are the highest. Following the $M A D$ matching procedure described above, we found a good solution for the realistic AGS 'Bleser' lattice, similar to what had been obtained by E.Courant on an idealized lattice. Since the real AGS is not too linear due to hysteresis in the magnets, with the non linearities represented in the model after work by Bleser, Auerbach, Tanaka and Luccio, the scaling of this low energy solution to higher energies proved an interesting task. Matching can only be achieved at the expense of a high value of the $\beta$ envelope function.

The good solution matches the insertion containing the snake to the rest of the AGS, keeping the $\beta$ functions unchanged, with respect to the bare AGS i.e. with no snake, everywhere but in the snake section. We will show the AGS $\beta$ later, together with the same obtained at higher energies.

\section{$5 \quad$ Matching and retuning at higher energies}

In the following, for each energy in unit of $\gamma$ we will give calculated values of quadrupole $K\left[1 / m^{2}\right]$, or currents $X[\mathrm{~A}]$, and gradients $G[T / m]$, where

$$
G=K \cdot B \rho, \quad K=-\frac{1}{B \rho} \frac{\partial B}{\partial r}=\frac{B}{B \rho L}, \quad B=\frac{\partial B}{\partial r}=c X+\text { higher order terms }
$$

Case 0: We first attempted to obtain matching conditions at increasing energies, up to AGS extraction: $\gamma=26$, using the same procedure than at injection, with the algorithms prodvided by the MAD program. The experience was very frustrating and inconclusive, because we could indeed find a solution at each individual energy, but these solutions did not yield monotonically decreasing curves for the matching quadrupole strength according what physical good sense would suggest. The problem was caused by the fact that the parameter space explored has 8 dimensions: 2 initial setting of the betatron values to initialize the matching, 4 values of the strength of the matching quadrupoles, and 2 values for the AGS tune quadrupoles. All parameters were very sensitive to the outcome. Results were found smooth and monotonic above a value of $\gamma=5$, but noisy and unreliable in the region between the good solution at injection and this energy. Here, we only show the orbit in these $M A D$ matched cases. Fig. 8 shows that there are clean 

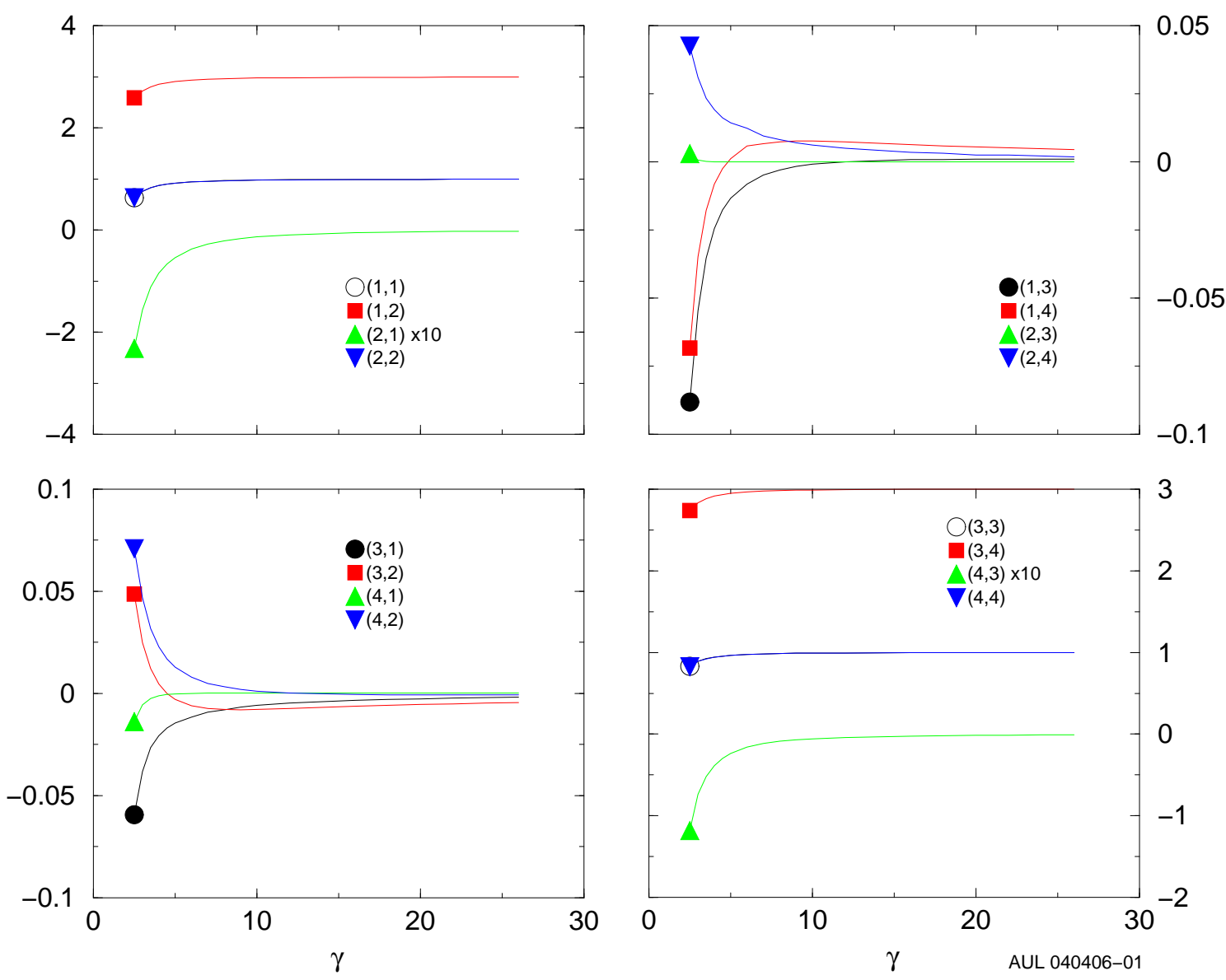

Figure 6: Elements of the transport matrix of the Snake. The upper left plot shows the elements for horizontal transfer, and the lower right plot for vertical transfer, respectively. The other two diagrams are for coupling elements. 


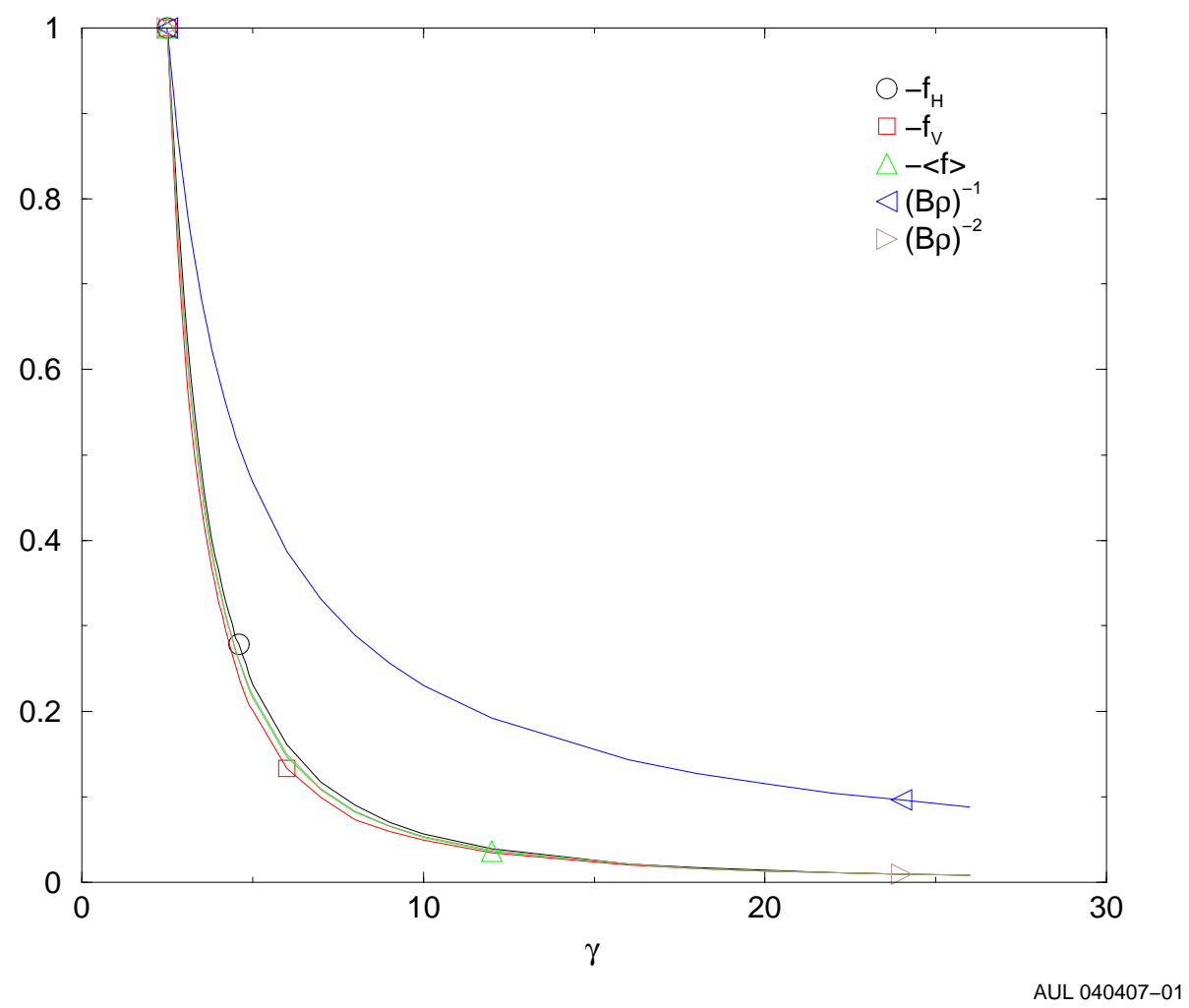

Figure 7: Focusing elements of the transport matrix of the Snake vs. beam energy. They scale as $1 /(B \rho)^{2}$. 
orbits outside the insertion not perturbed with what is obtained w/o the snake. We don't show the corresponding matching quadrupole currents, because they are of no practical use.

Case 1: Using the solution found at injection as a solid base, consistent results were found instead by simply scaling the matching quadrupole strengths K like the focusing elements in the transfer matrices of Fig. 7

$$
K=K_{0} \frac{(B \rho)_{i n j}^{2}}{(B \rho)^{2}},
$$

A first run was done by starting from a stable value of the betatron tune $\nu_{x}=8.60$ and $\nu_{y}=8.90$, for the AGS with the snake inserted. The high value of the vertical tune was dictated by a desire to have it as close as possible to the integer for spin preservation (in a window of tune to avoid a snake resonance). The starting was the good case referred to above. Then, we proceeded scaling down the matching quads as said, and retuning at each step to obtain the "magic" value $\nu_{y}=8.96$. The horizontal value of the betatron tune was left free. The purpose was to find the values of $\nu_{x}$ vs. energy that the lattice liked most. Fit of these values with some analytical expression could suggest how to proceed further. Results of this run are shown in Figs. 9, 10, 11, 12, and 13.

The figures show the following:

- A continuous acceptable solution is found at all energies. The matching quadrupoles fade away at high energy, where the snake has very little residual focusing.

- Rematching in order to obtain a high value of the verical betatron tune requires large currents (the AGS tune quads power supply is rated for $700 \mathrm{~A}$ maximum current, so we are within the limits).

- A Best fit of the horizontal tune found by the $M A D$ matching algorithm is an inverse-energy square law

$$
\nu_{x} \approx 8.54457+1.85346 / \gamma^{2}
$$

- At low energy the beam is wide in vertical. At high energy the beam is wider in the horizontal direction. The two trends exchange roles at about $\gamma=5$, where we found difficulties when trying to obtain an optimum match. The cause is complex, because is due to the interplay of the focusing properties of the bare lattice, almost constant over the entire energy range and the focusing of the helical snake that decreases with energy.

Case 2: Using the "natural" trend of the vertical tune of Case 1, we run a Case 2, that used the same values of the matching quadrupoles vs. energy, but wehre we searched for a rematch with $\nu_{y}$ still kept at a value of 8.96, but with $\nu_{y}$ made to follow the fit of Eq.(1). Results are shown in Figs. 14, 15, 16, and 17. For this case we still obtain a noisy value of the orbit amplitude at low energy. Tables 1 and 2 give numerical values for this case

Case 3: Can we keep a low constant value of $\nu_{x}$ along the acceleration? a value of 8.60 , combined with 8.96 for the vertical proved impossible. A value $\nu_{x}=8.64$ did converge (Case 3 ), for $\gamma>4$, but fell in a region of instability below. Results for the tune, the maximum beta, and the corresponding values of tune quads parameters are shown in Figs. 18, 19, 20, and 21, respectively.

The complete beta wave and dispersion for 4 chosen energies for Case 2 are shown in Figs. 22 and 23 

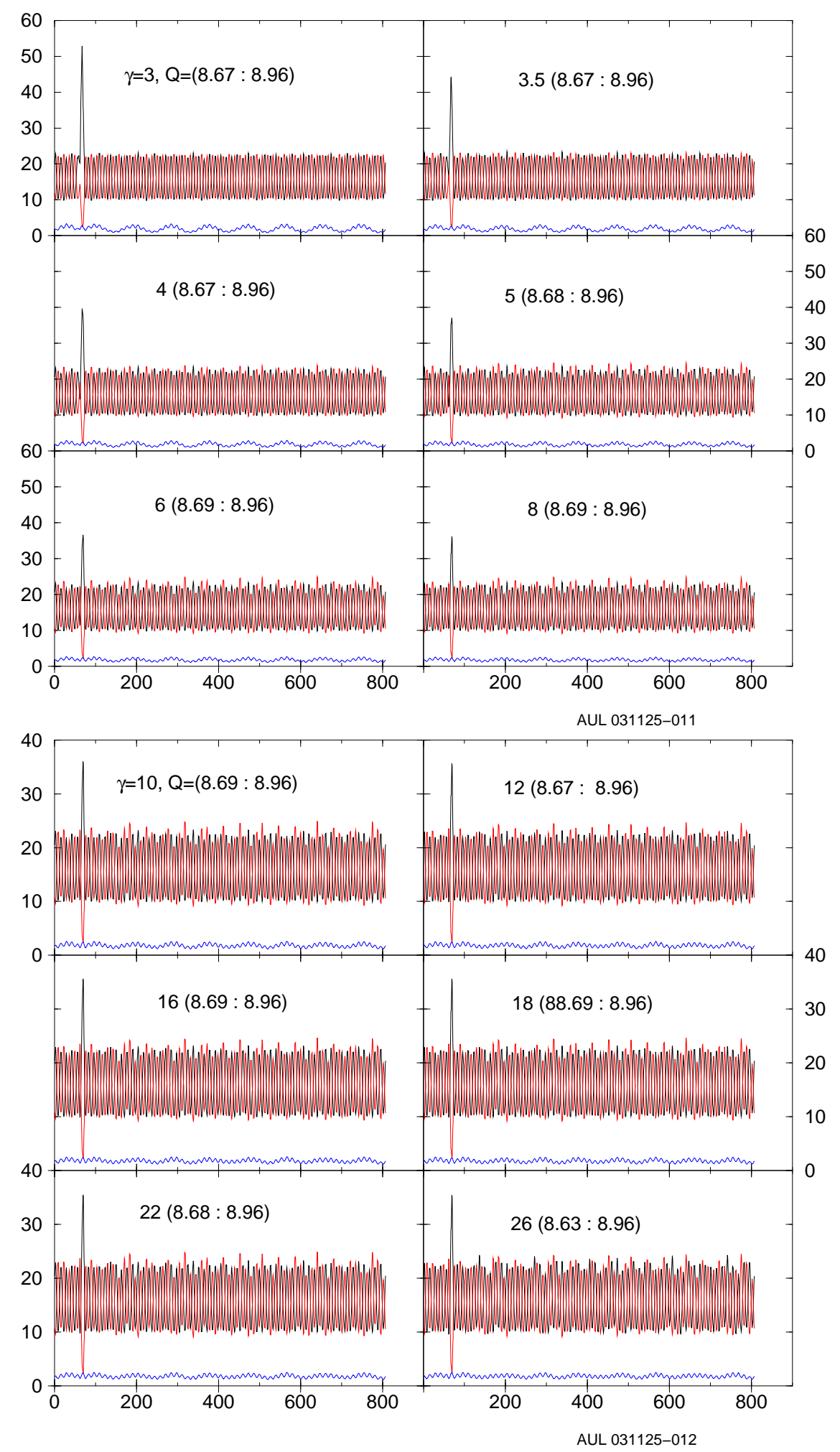

Figure 8: Bleser lattice: $\gamma$ from 1.5 to 26. MAD optimization of matching 


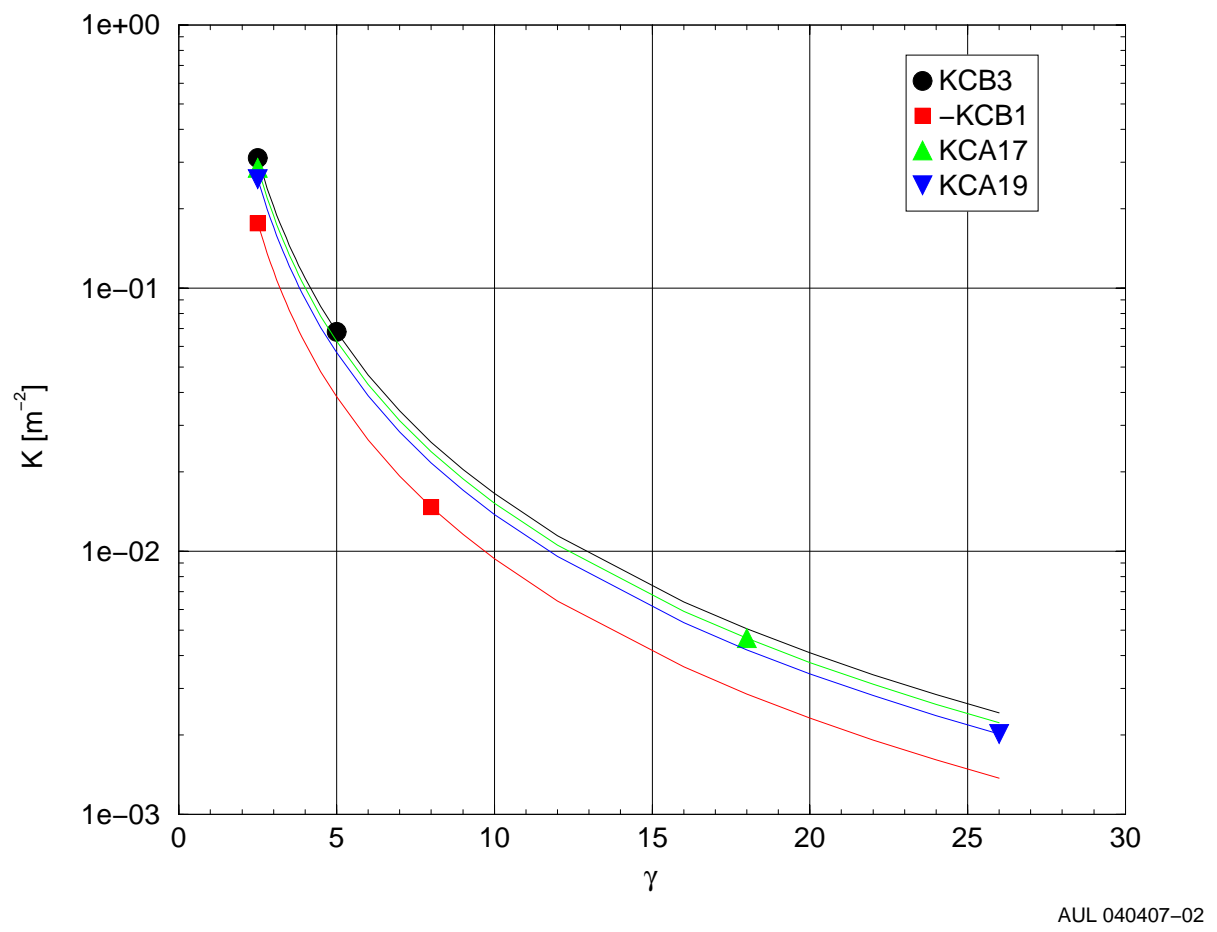

Figure 9: $K$ parameter of the matching quadrupoles as a function of energy. The inverse- $(B \rho)^{2}$ law is assumed.

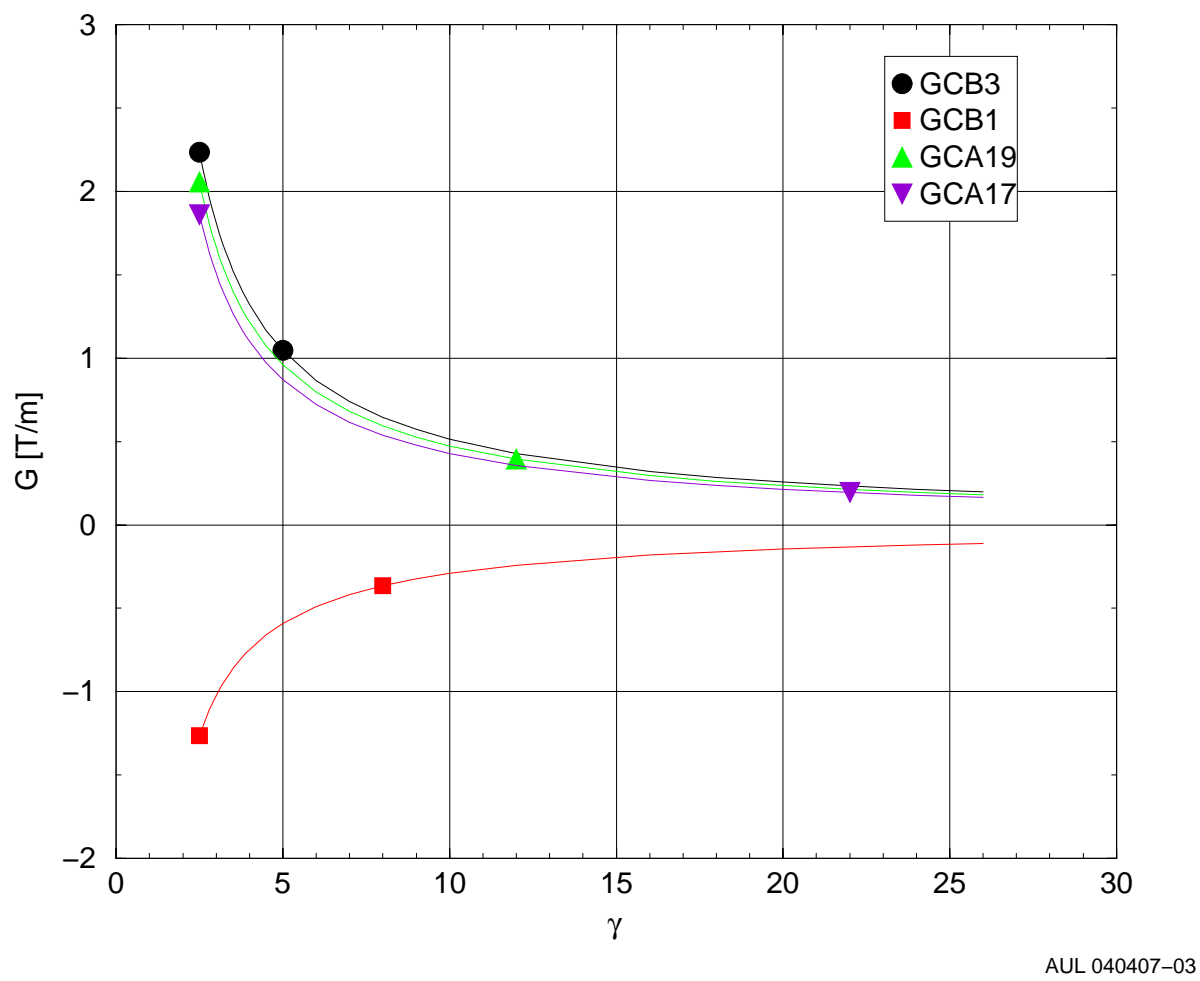

Figure 10: Gradient of the matching quadrupoles. 


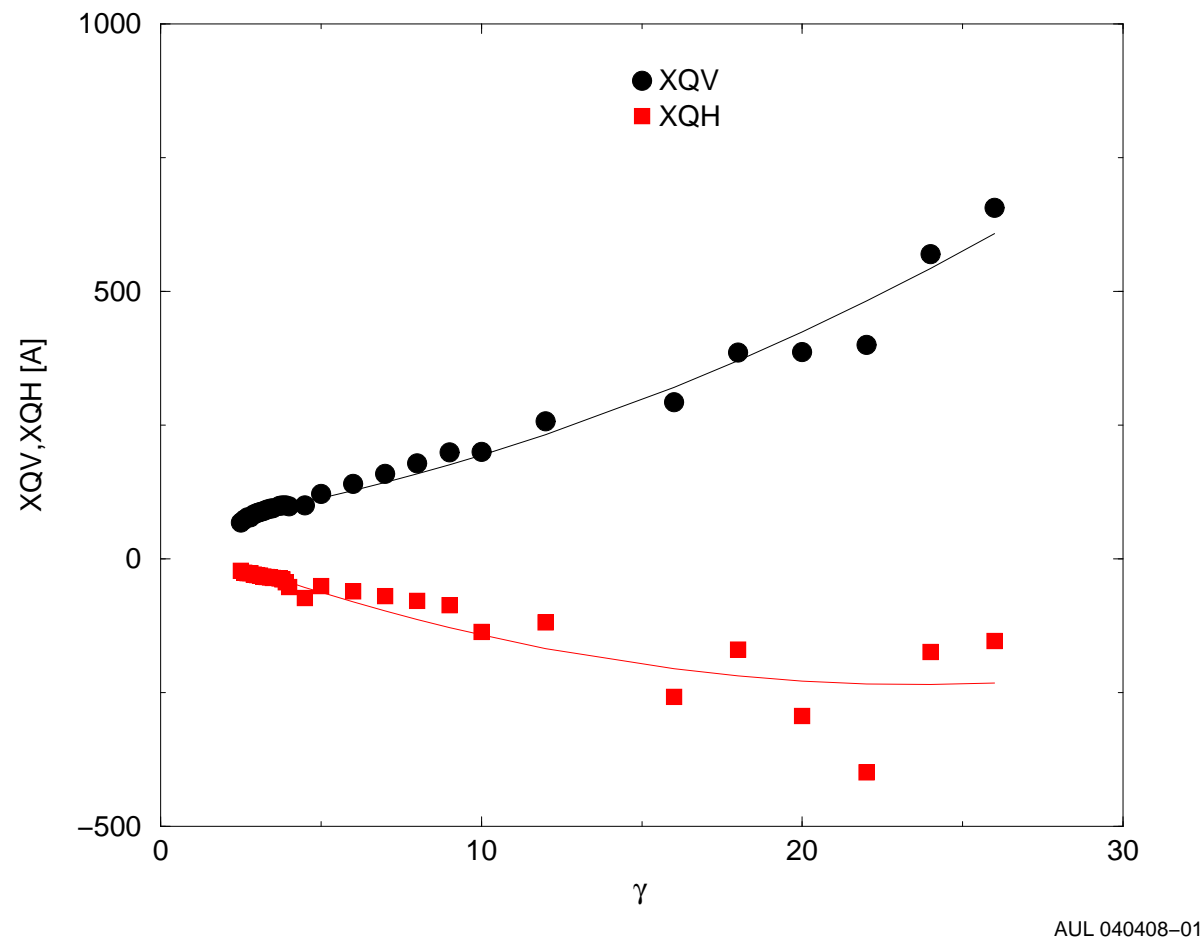

Figure 11: Case 1. Current in the tune quadrupoles [A].

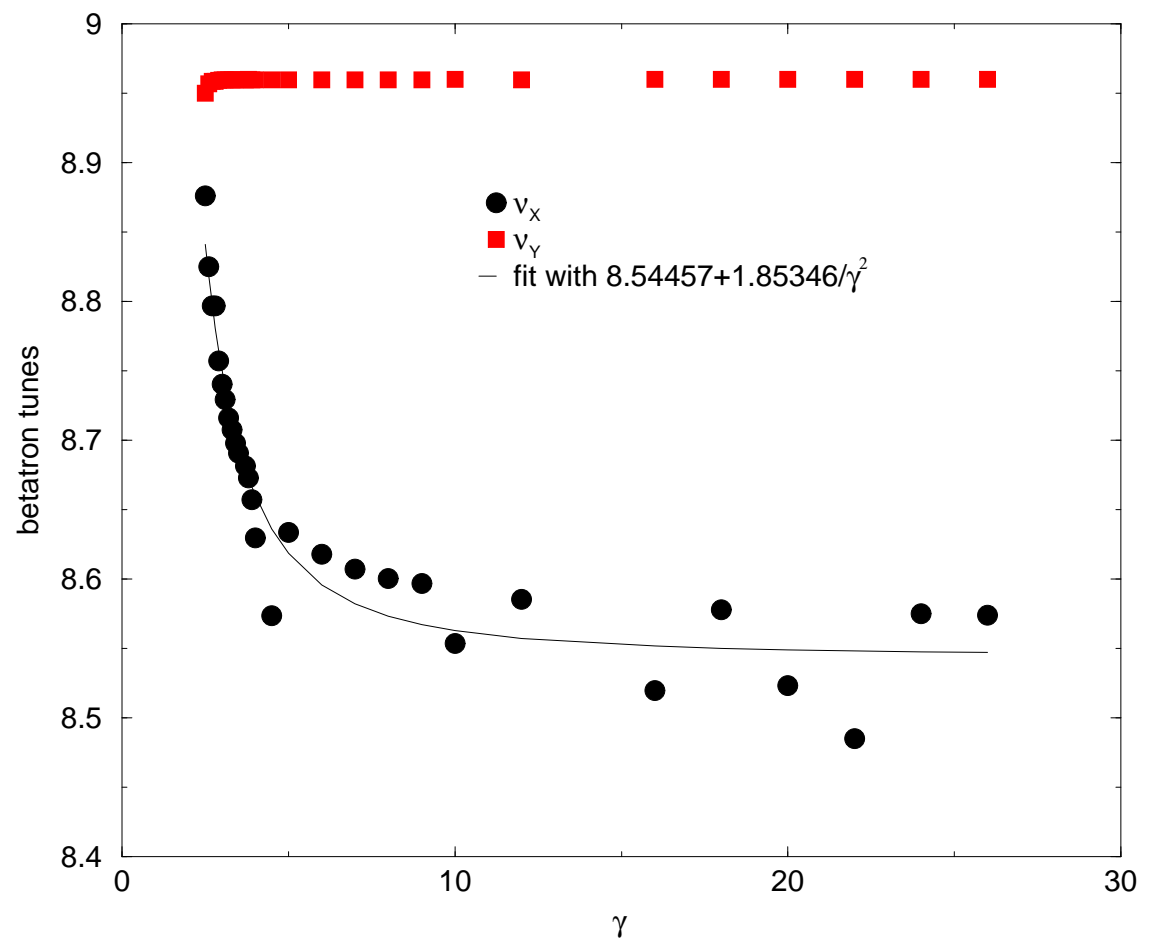

AUL 040408-03

Figure 12: Case 1: Betatron tunes. Retune is done to obtain $\nu_{y}=8.96 . \nu_{x}$ is left free. 


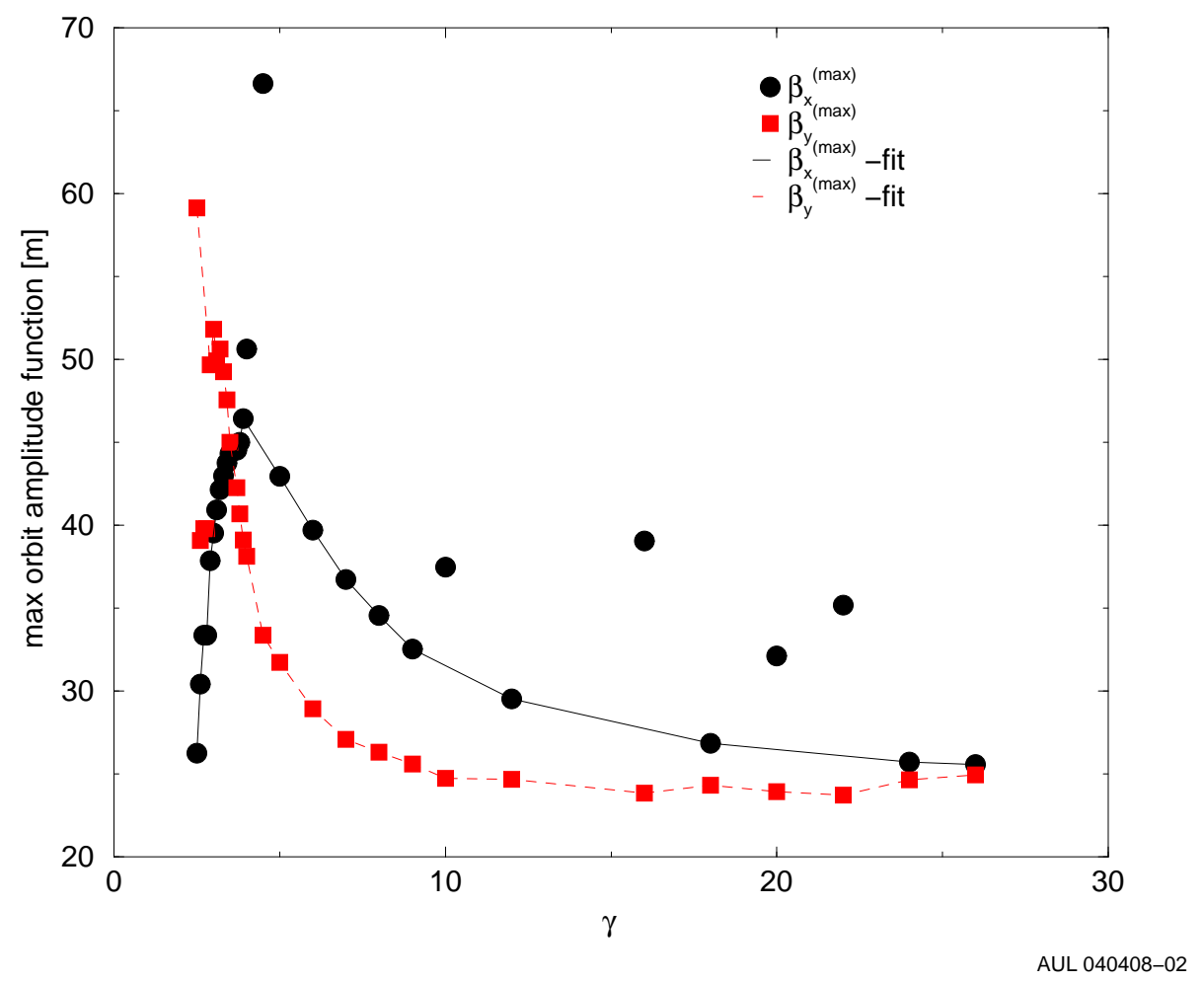

Figure 13: Case1: Maximum value of the beam amplitude function $\beta$ after matching and retuning.

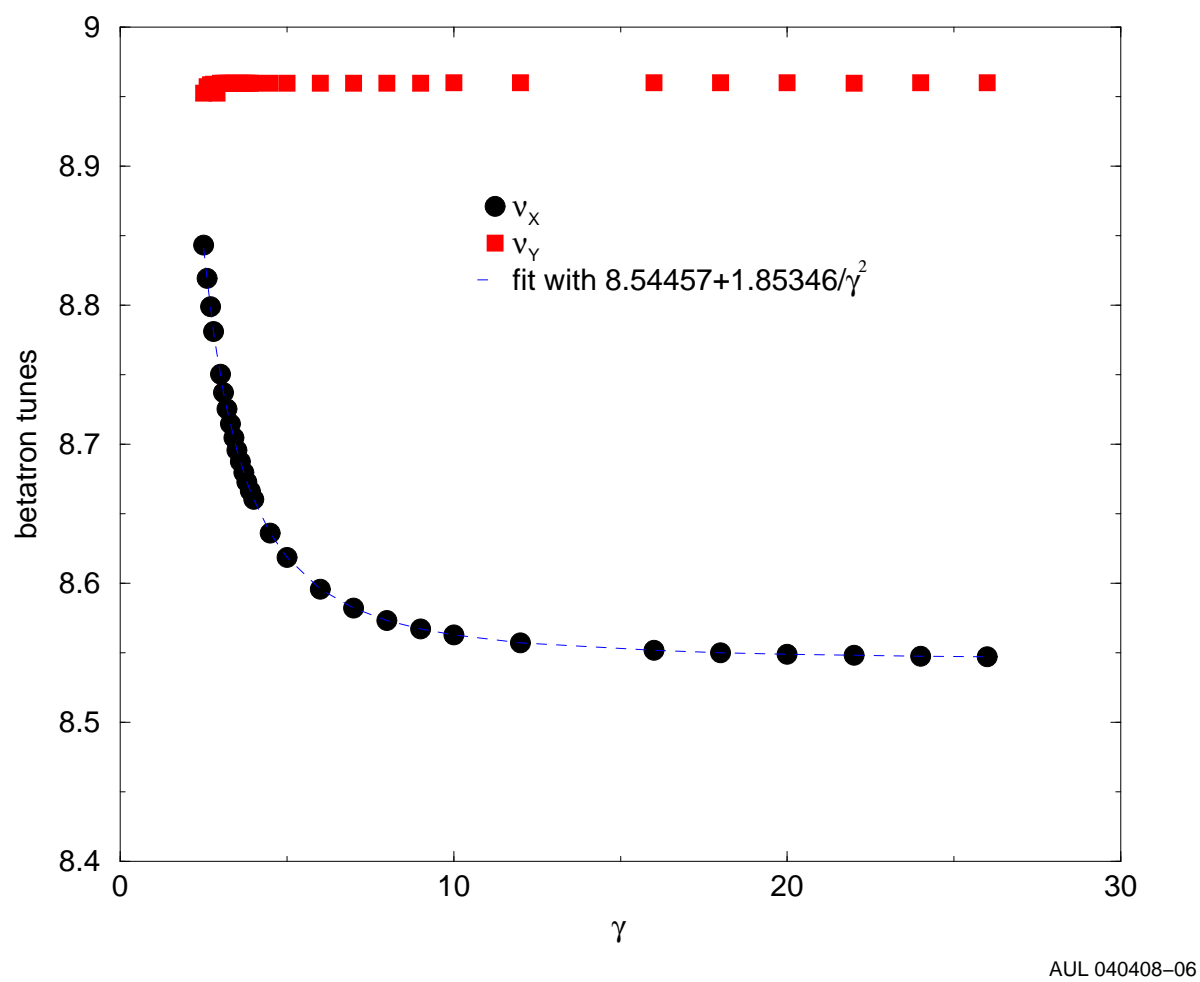

Figure 14: Case 2. Betatron tunes after rematch. 


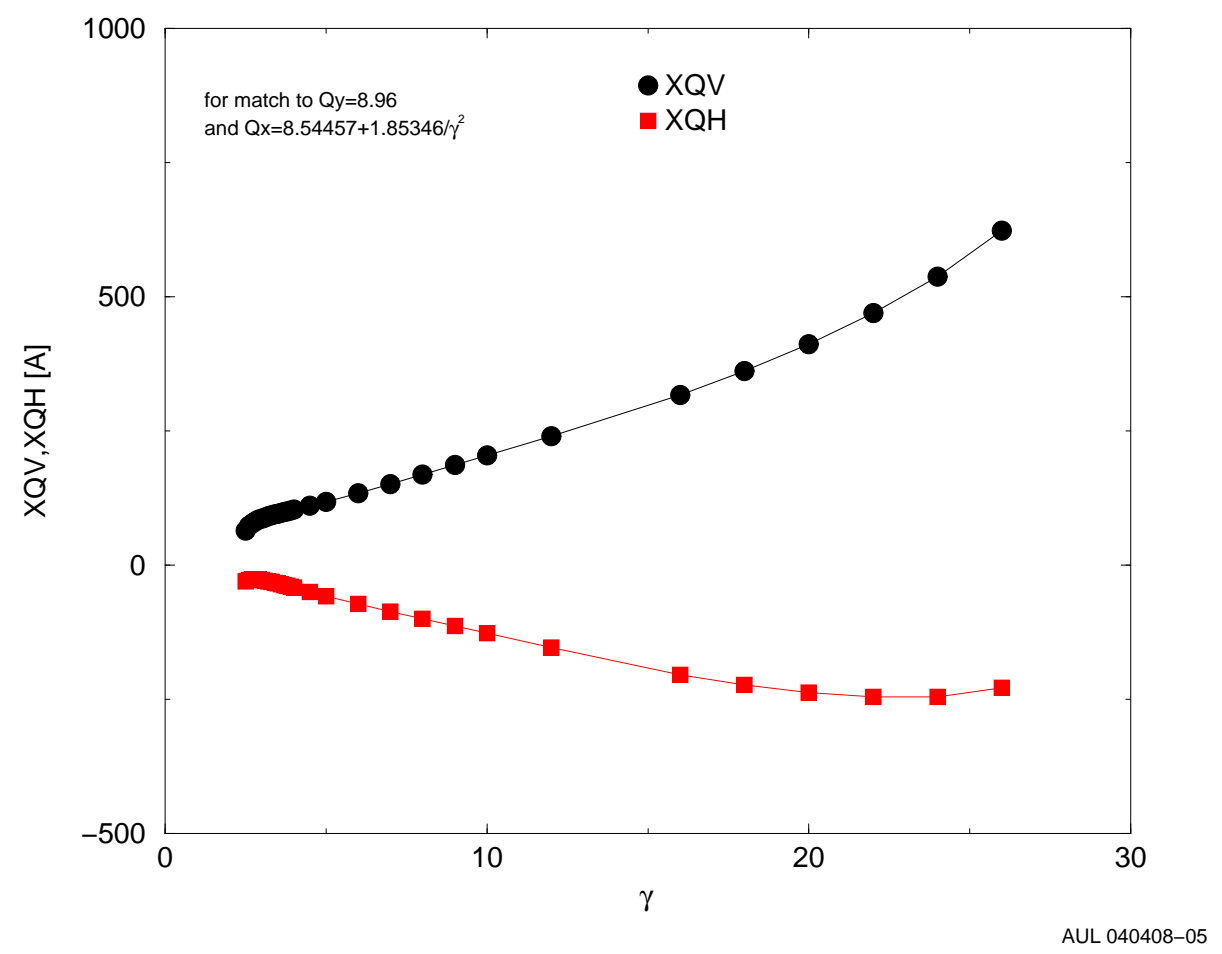

Figure 15: Case 2. Current in the tune quadrupoles.

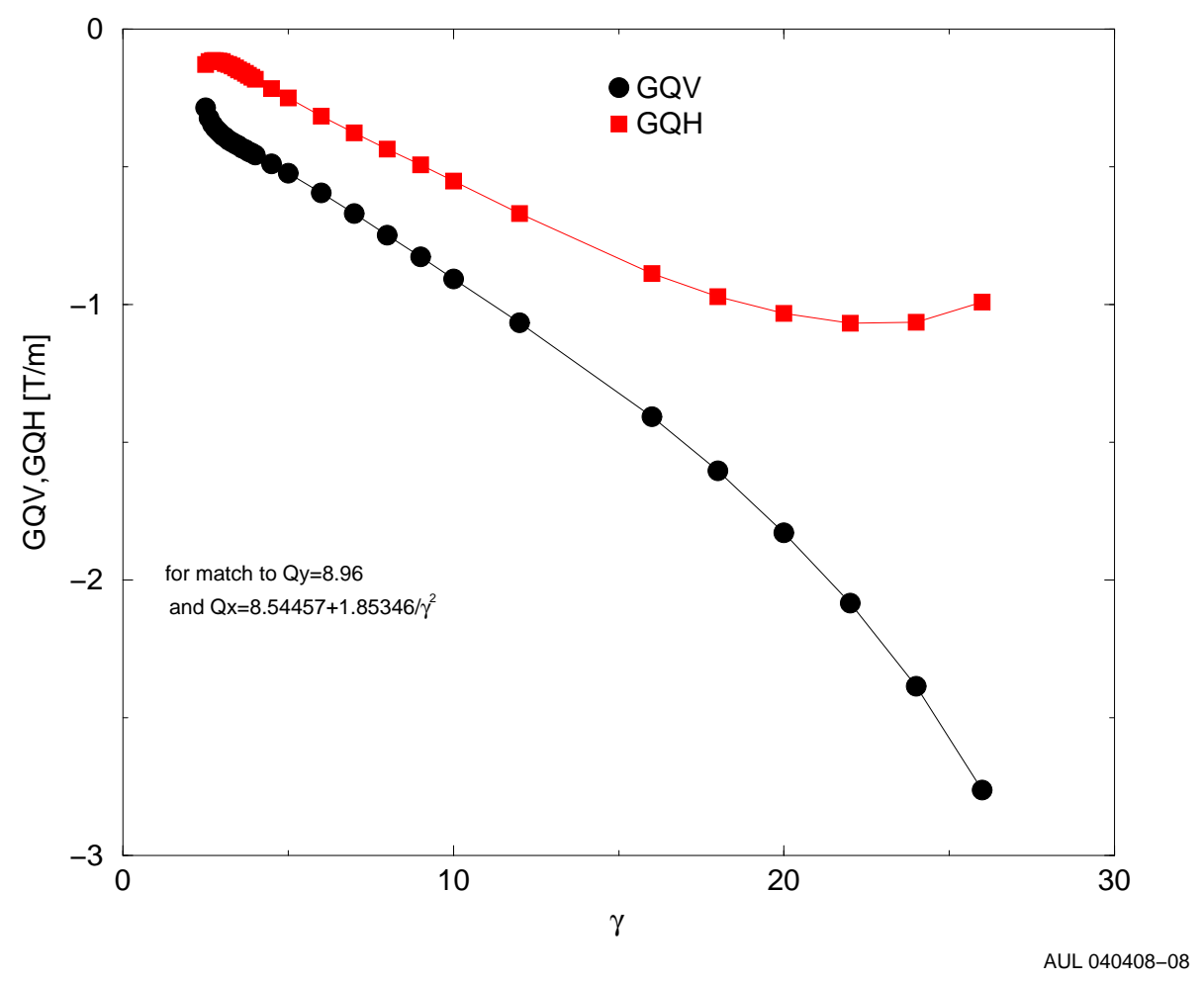

Figure 16: Case 2. gradients in the tune quadrupoles. 


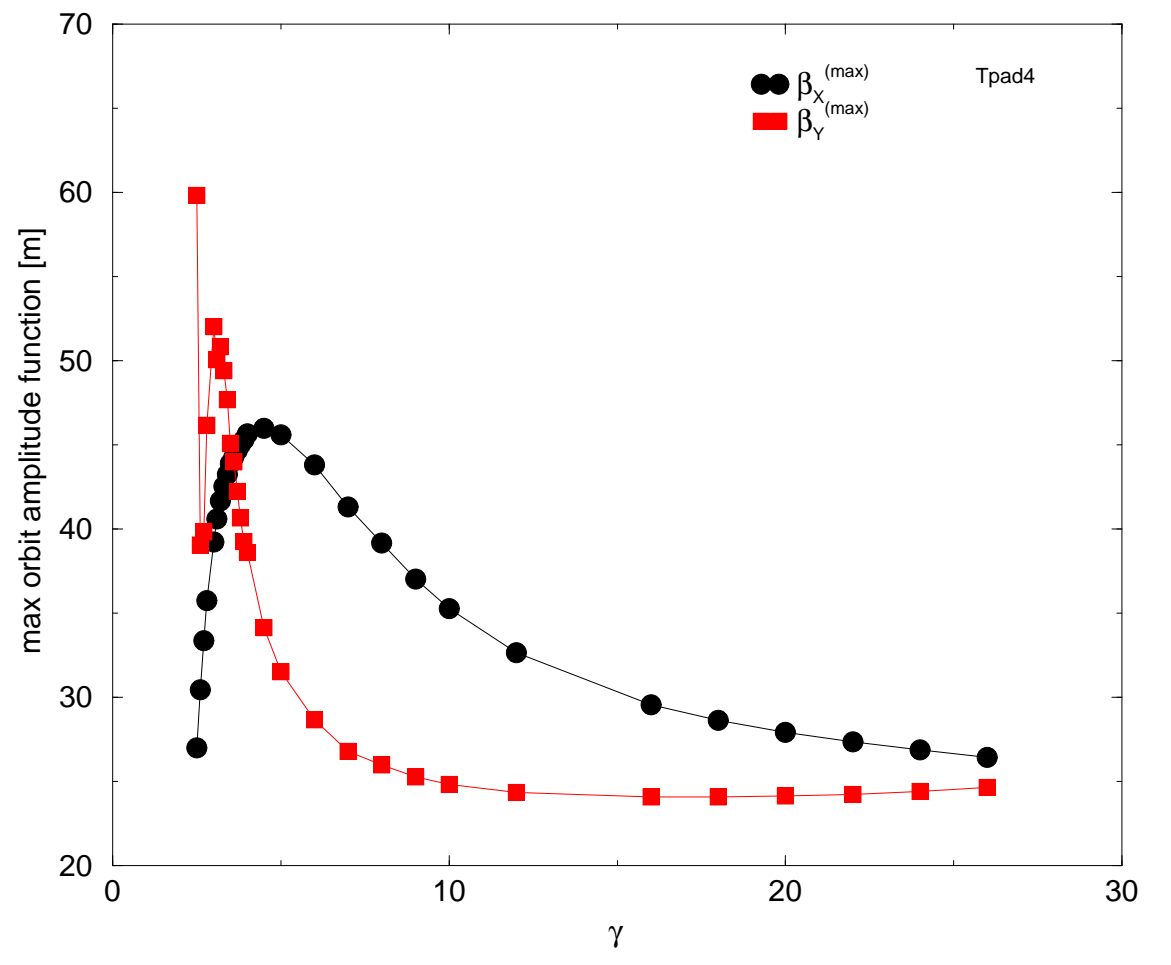

AUL 040408-07

Figure 17: Case 2. Maximum beta.

Table 1: Case 2: Quadrupole strengths to match snake to AGS lattice

\begin{tabular}{rrrrrrrrr}
\hline$\gamma$ & KCB3 & KCB1 & KCA19 & KCA17 & GCB3 & GCB1 & GCA19 & GCA17 \\
\hline 2.5 & $0.3114602-$ & 0.1761206 & 0.2864219 & 0.2593226 & 2.2335215 & -1.2629838 & 2.0539690 & 1.8596362 \\
3.0 & $0.2043954-$ & 0.1155790 & 0.1879641 & 0.1701802 & 1.8093592 & -1.0231338 & 1.6639051 & 1.5064775 \\
3.5 & $0.1453479-$ & 0.0821895 & 0.1336634 & 0.1210170 & $1.5257856-$ & 0.8627822 & 1.4031280 & 1.2703734 \\
4.0 & $0.1090109-$ & 0.0616421 & 0.1002475 & 0.0907628 & $1.3213691-$ & 0.7471913 & 1.2151445 & 1.1001756 \\
4.5 & $0.0849435-$ & 0.0480328 & 0.0781149 & 0.0707242 & $1.1664185-$ & 0.6595718 & 1.0726503 & 0.9711633 \\
5.0 & $0.0681318-$ & 0.0385263 & 0.0626547 & 0.0567267 & $1.0446340-$ & 0.5907066 & 0.9606560 & 0.8697652 \\
6.0 & $0.0467189-$ & 0.0264180 & 0.0429632 & 0.0388983 & $0.8650391-$ & 0.4891515 & 0.7954988 & 0.7202340 \\
7.0 & $0.0340659-$ & 0.0192631 & 0.0313273 & 0.0283633 & $0.7386678-$ & 0.4176926 & 0.6792864 & 0.6150168 \\
8.0 & $0.0259549-$ & 0.0146767 & 0.0238684 & 0.0216101 & $0.6447621-$ & 0.3645920 & 0.5929298 & 0.5368307 \\
9.0 & $0.0204395-$ & 0.0115579 & 0.0187964 & 0.0170180 & $0.5721696-$ & 0.3235433 & 0.5261730 & 0.4763900 \\
10. & $0.0165168-$ & 0.0093397 & 0.0151890 & 0.0137519 & $0.5143422-$ & 0.2908438 & 0.4729943 & 0.4282427 \\
12. & $0.0114347-$ & 0.0064659 & 0.0105154 & 0.952057 & $0.4279586-$ & 0.2419966 & 0.3935550 & 0.3563195 \\
16. & $0.0064124-$ & 0.0036260 & 0.0058969 & 0.0053389 & $0.3204790-$ & 0.1812205 & 0.2947158 & 0.2668317 \\
18. & $0.0050624-$ & 0.0028626 & 0.0046554 & 0.0042149 & $0.2847531-$ & 0.1610186 & 0.2618618 & 0.2370862 \\
20. & $0.0040981-$ & 0.0023173 & 0.0037687 & 0.0034121 & $0.2562024-$ & 0.1448741 & 0.2356064 & 0.2133149 \\
22. & $0.0033854-$ & 0.0019143 & 0.0031132 & 0.0028187 & $0.2328607-$ & 0.1316751 & 0.2141410 & 0.1938804 \\
24. & $0.0028437-$ & 0.0016080 & 0.0026151 & 0.0023677 & $0.2134203-$ & 0.1206822 & 0.1962635 & 0.1776943 \\
26. & $0.0024224-$ & 0.0013698 & 0.0022277 & 0.0020169 & $0.1969780-$ & 0.1113847 & 0.1811430 & 0.1640045 \\
\hline
\end{tabular}


Table 2: Case 2: Tune quadrupole strengths to retune AGS lattice. Eq.(1)

\begin{tabular}{rrrrrrrrr}
\hline$\gamma$ & XQV & XQV & GQV & GQH & Qx & Qy & $\beta_{x}^{\max }$ & $\beta_{y}^{\max }$ \\
\hline 2.5 & 64.08840 & -30.60576 & -0.2870466 & -0.1299278 & 8.843231 & 8.952803 & 26.997752 & 59.824393 \\
3.0 & 86.53432 & -28.19026 & -0.3862544 & -0.1193275 & 8.750545 & 8.959682 & 39.250029 & 52.042858 \\
3.5 & 95.28881 & -34.80679 & -0.4249795 & -0.1483590 & 8.695878 & 8.959925 & 43.925902 & 45.116546 \\
4.0 & 102.75767 & -42.63781 & -0.4580305 & -0.1826991 & 8.660413 & 8.959974 & 45.674643 & 38.601271 \\
4.5 & 110.02215 & -50.60077 & -0.4901880 & -0.2175951 & 8.636100 & 8.959989 & 45.991600 & 34.149593 \\
5.0 & 117.62807 & -58.38337 & -0.5238678 & -0.2516783 & 8.618709 & 8.959994 & 45.621745 & 31.535574 \\
6.0 & 133.82942 & -73.17413 & -0.5956438 & -0.3163912 & 8.596056 & 8.959998 & 43.837995 & 28.676076 \\
7.0 & 150.66591 & -87.11228 & -0.6702787 & -0.3772988 & 8.582397 & 8.959999 & 41.325345 & 26.812699 \\
8.0 & 168.44222 & -100.67960 & -0.7491229 & -0.4365156 & 8.573531 & 8.959999 & 39.171065 & 26.007628 \\
9.0 & 186.20909 & -113.99635 & -0.8279628 & -0.4945713 & 8.567453 & 8.959999 & 37.028425 & 25.283354 \\
10. & 204.11551 & -127.38189 & -0.9074532 & -0.5528604 & 8.563105 & 8.960000 & 35.275679 & 24.849346 \\
12. & 240.08177 & -154.38257 & -1.0671842 & -0.6702406 & 8.557441 & 8.960000 & 32.669800 & 24.372170 \\
16. & 316.64004 & -204.51250 & -1.4073205 & -0.8875331 & 8.551810 & 8.960000 & 29.575014 & 24.087135 \\
18. & 361.01844 & -224.06799 & -1.6044666 & -0.9721115 & 8.550291 & 8.960000 & 28.631608 & 24.102803 \\
20. & 411.52746 & -238.29043 & -1.8287671 & -1.0335751 & 8.549204 & 8.960002 & 27.930254 & 24.155958 \\
22. & 469.15718 & -246.38287 & -2.0845574 & -1.0685328 & 8.548354 & 8.959956 & 27.353523 & 24.254967 \\
24. & 537.06423 & -245.56874 & -2.3857901 & -1.0650163 & 8.547788 & 8.960002 & 26.881445 & 24.408383 \\
26. & 622.42157 & -228.62447 & -2.7642171 & -0.9918067 & 8.547272 & 8.960021 & 26.458312 & 24.645137 \\
\hline
\end{tabular}

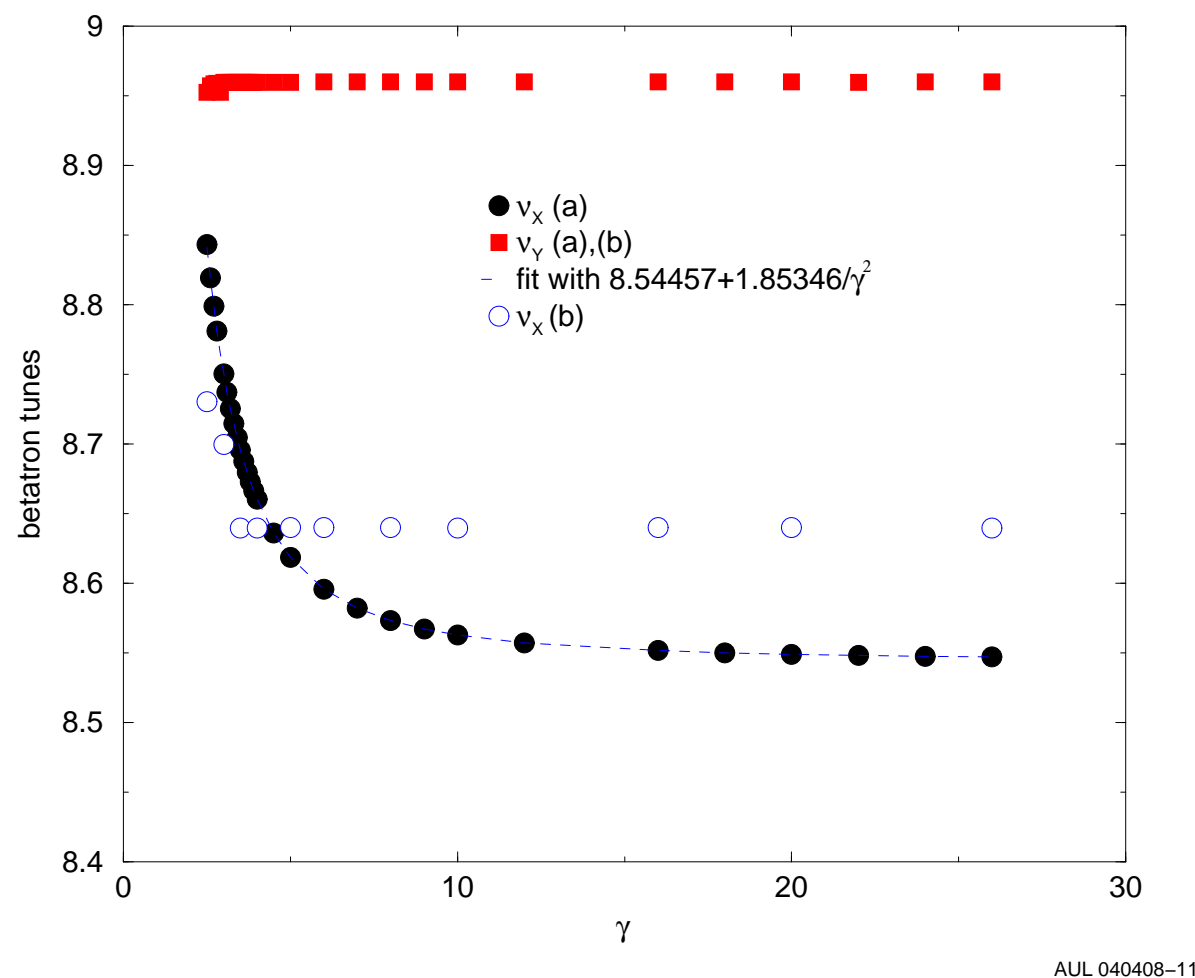

Figure 18: Case 3. Betatron tunes after rematch, compared with Case 2. 


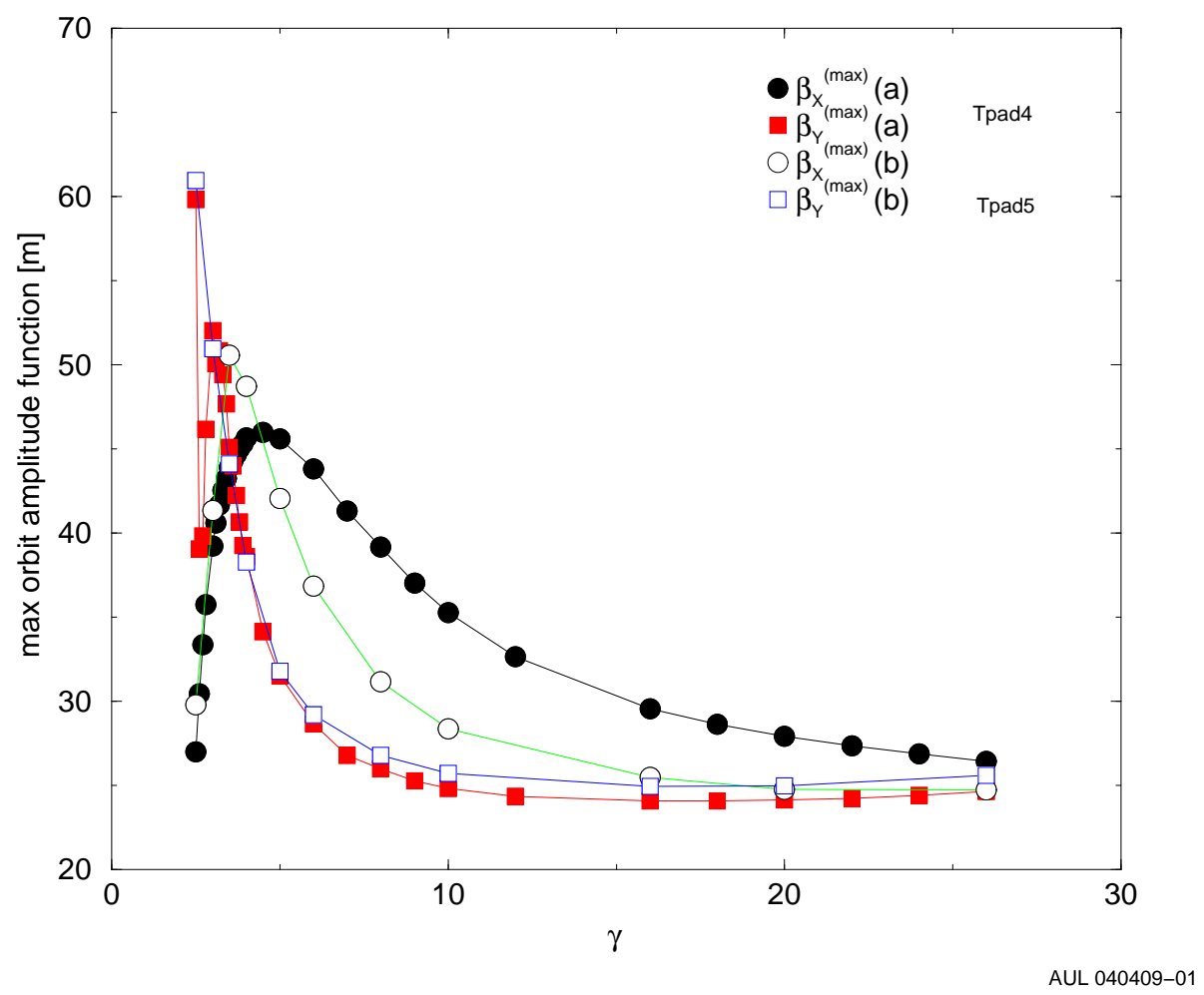

Figure 19: Case 3. Maximum beta. Compared with Case 2.

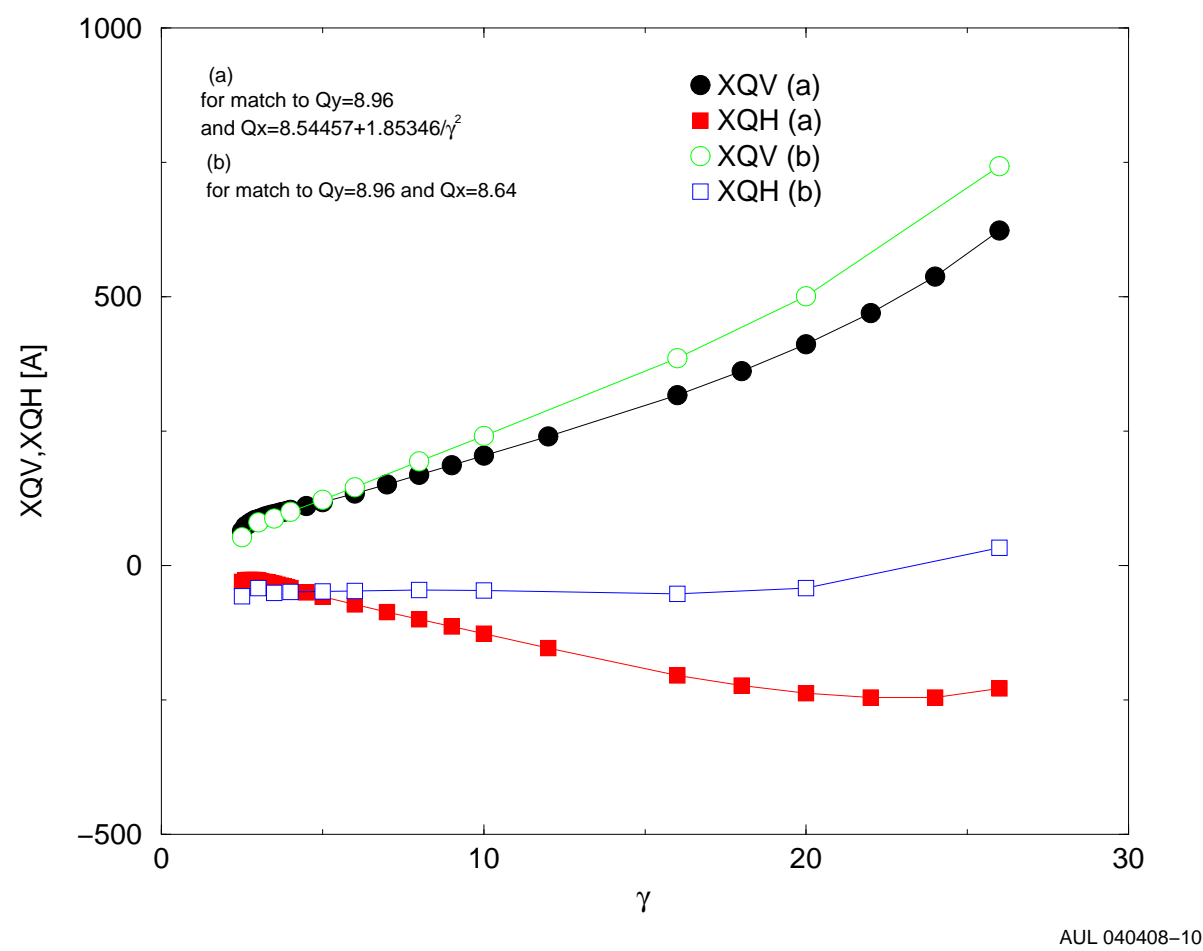

Figure 20: Case 3. Current in the tune quadrupoles. Compared with Case 2. 


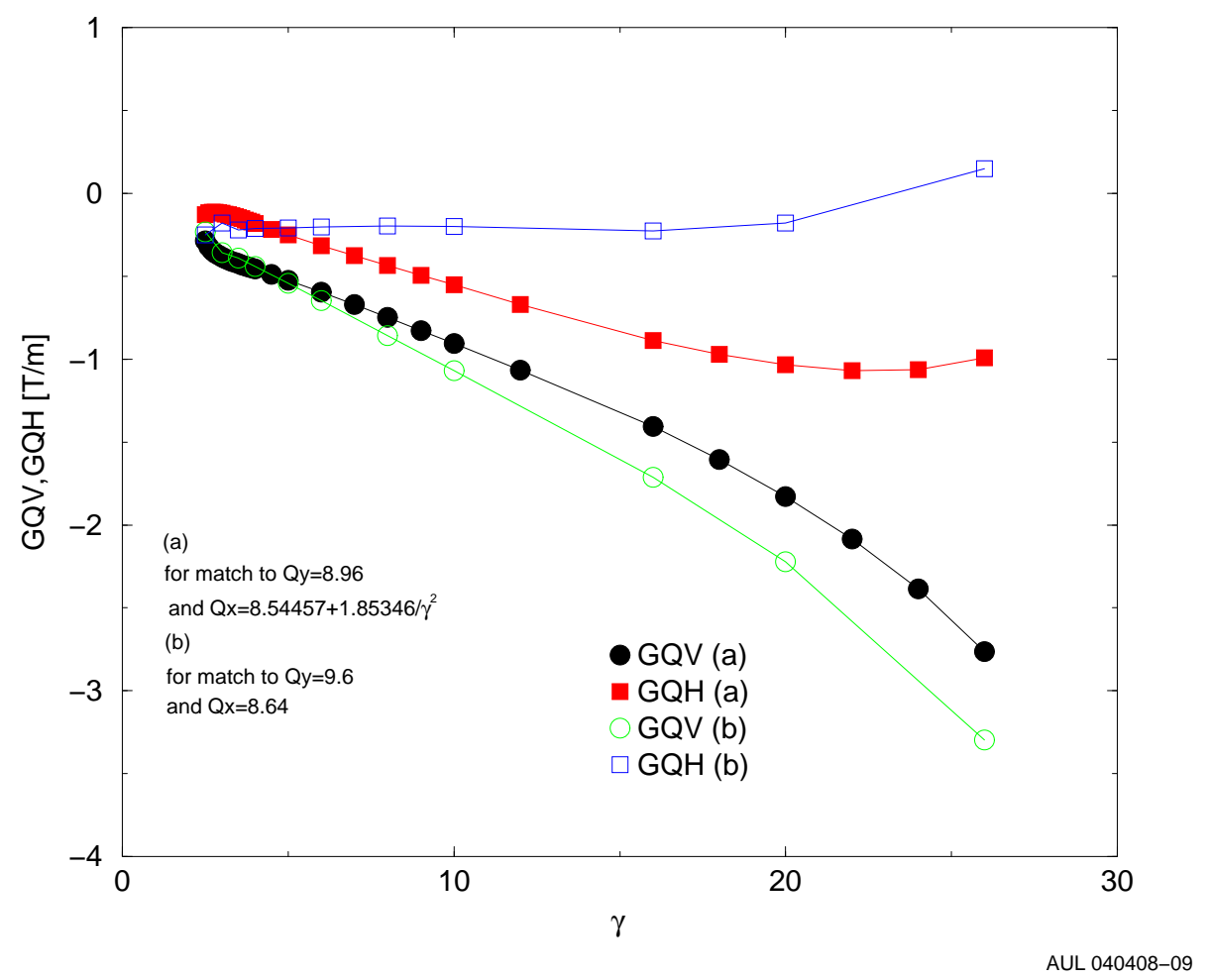

Figure 21: Case 3. gradients in the tune quadrupoles. Compared with Case 2. 

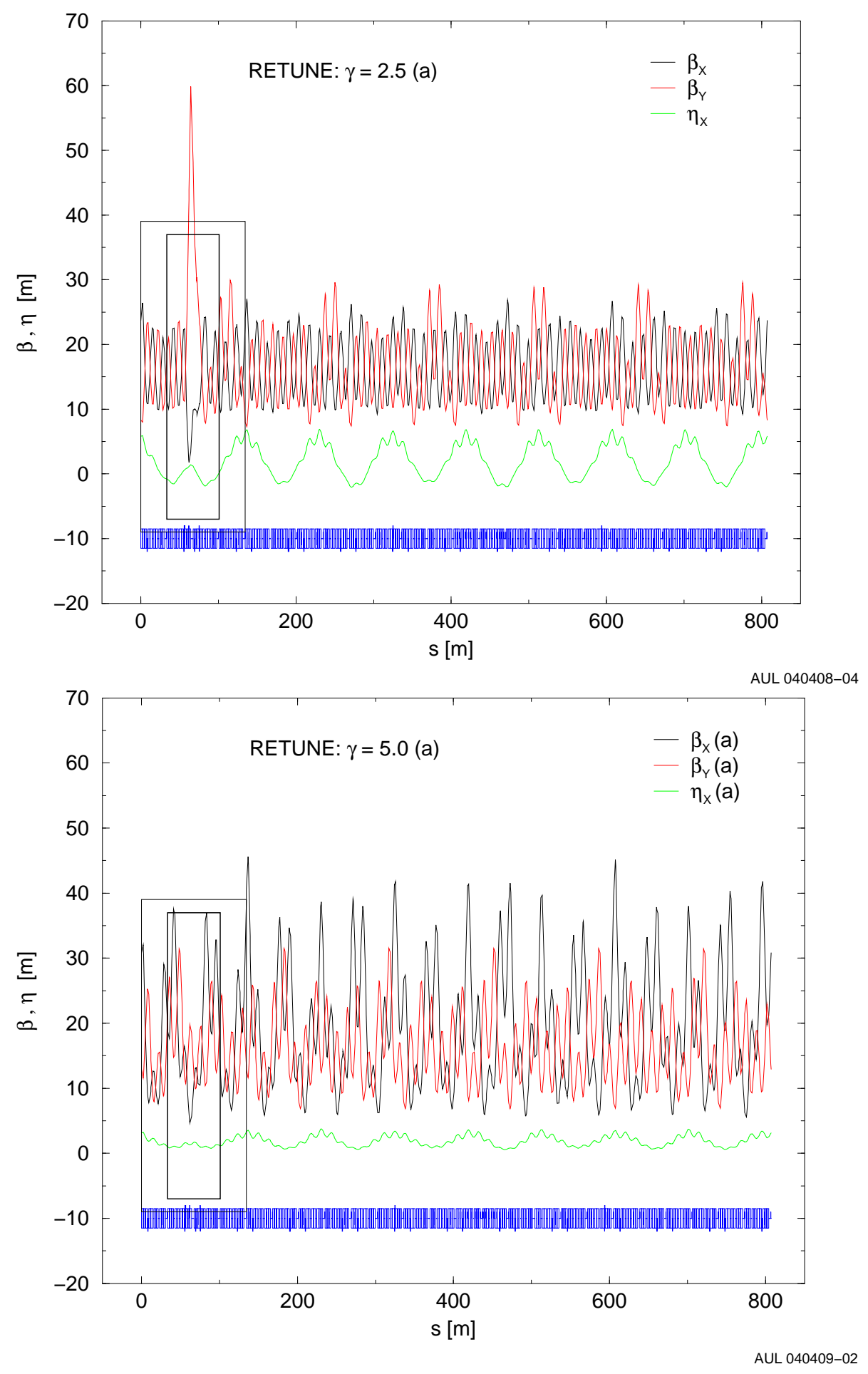

Figure 22: Case 2. Beta wave and dispersion at two chosen energies. 

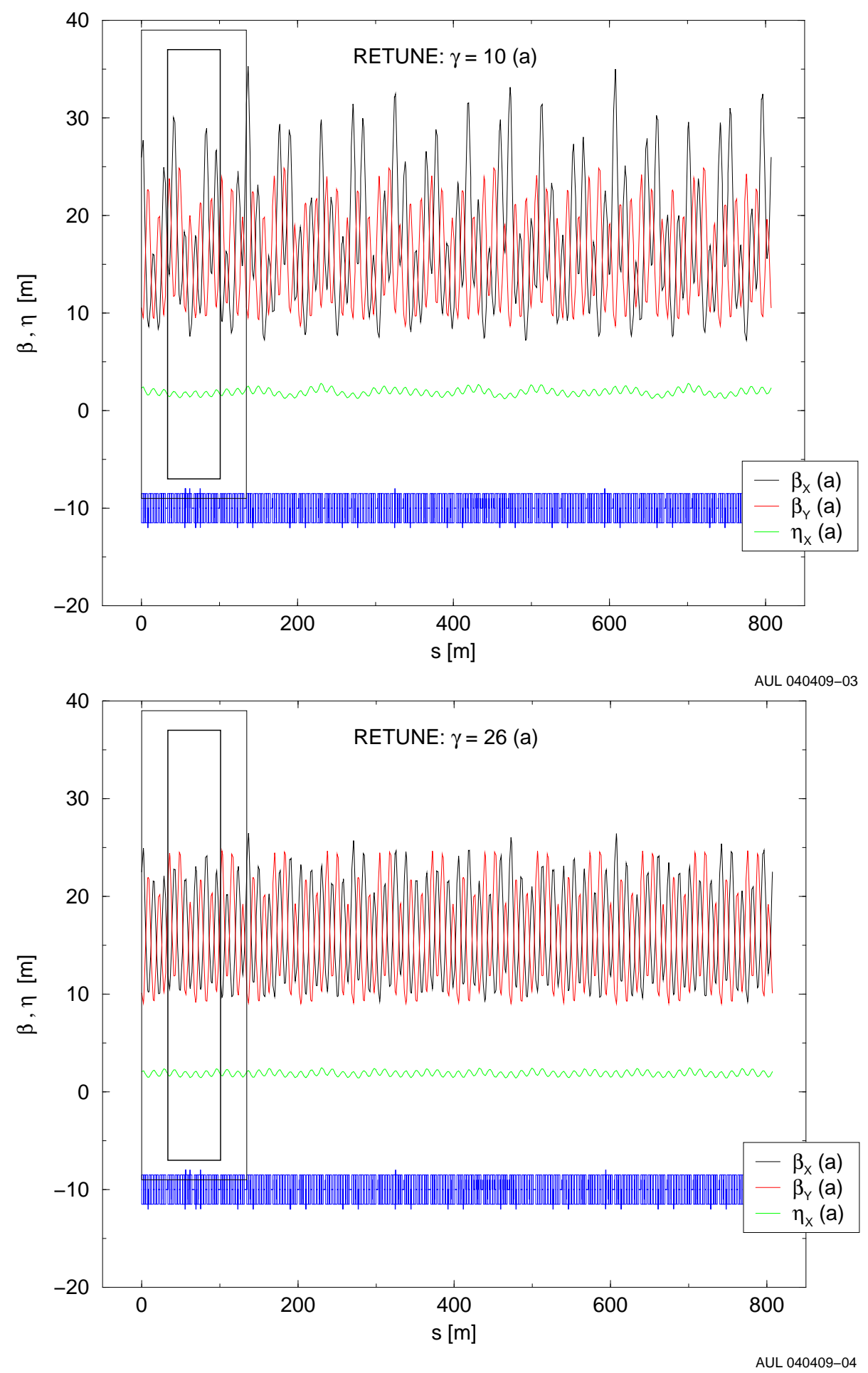

Figure 23: Case 2. Beta wave and dispersion at two chosen energies. 


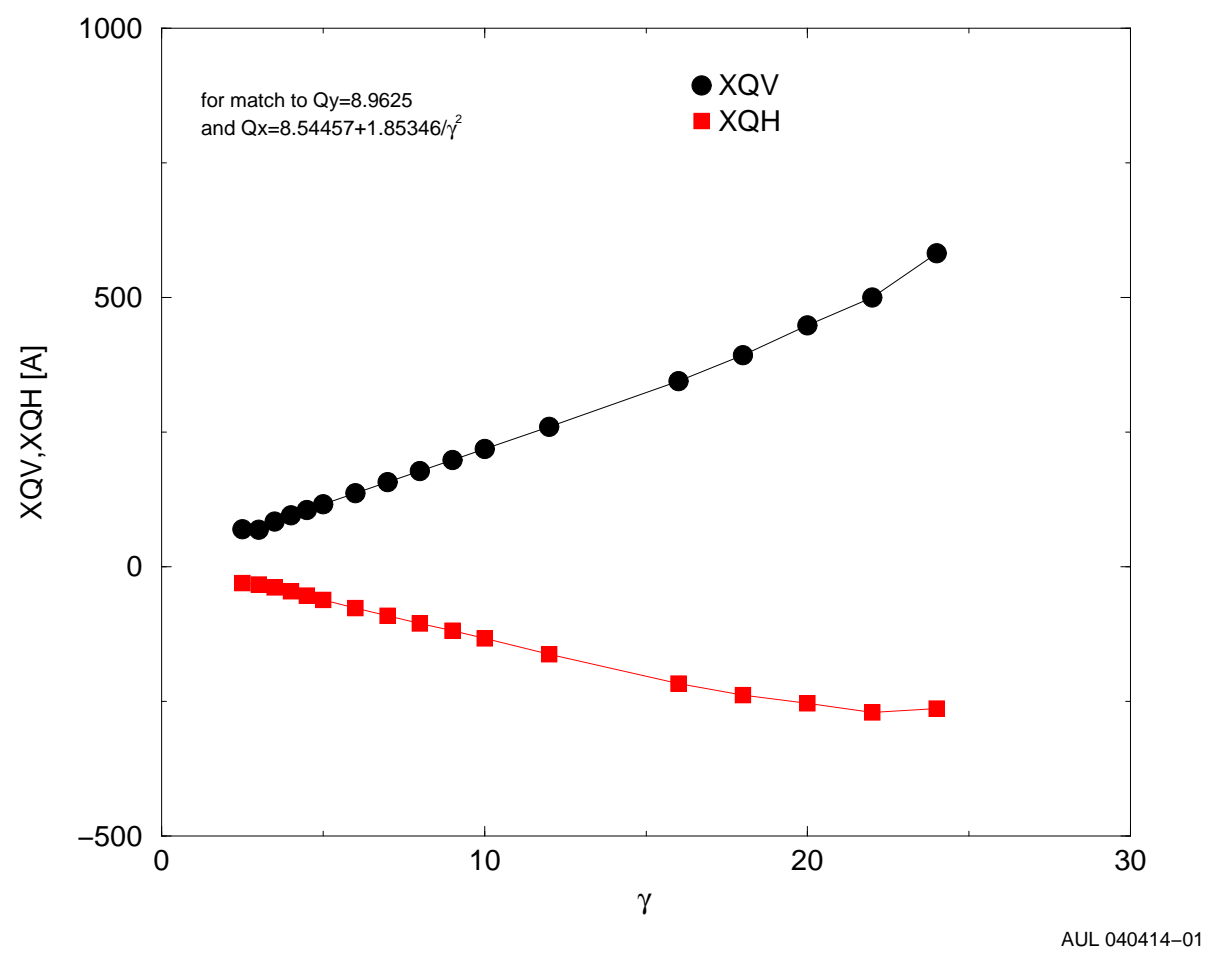

Figure 24: Case 4: current in the tune quadrupoles.

Case 4 Matching quadrupole A17 is lumped with tune quad QH, and B3 with QV, respectively. Retune to obtain $\nu_{y}=8.9625$. 


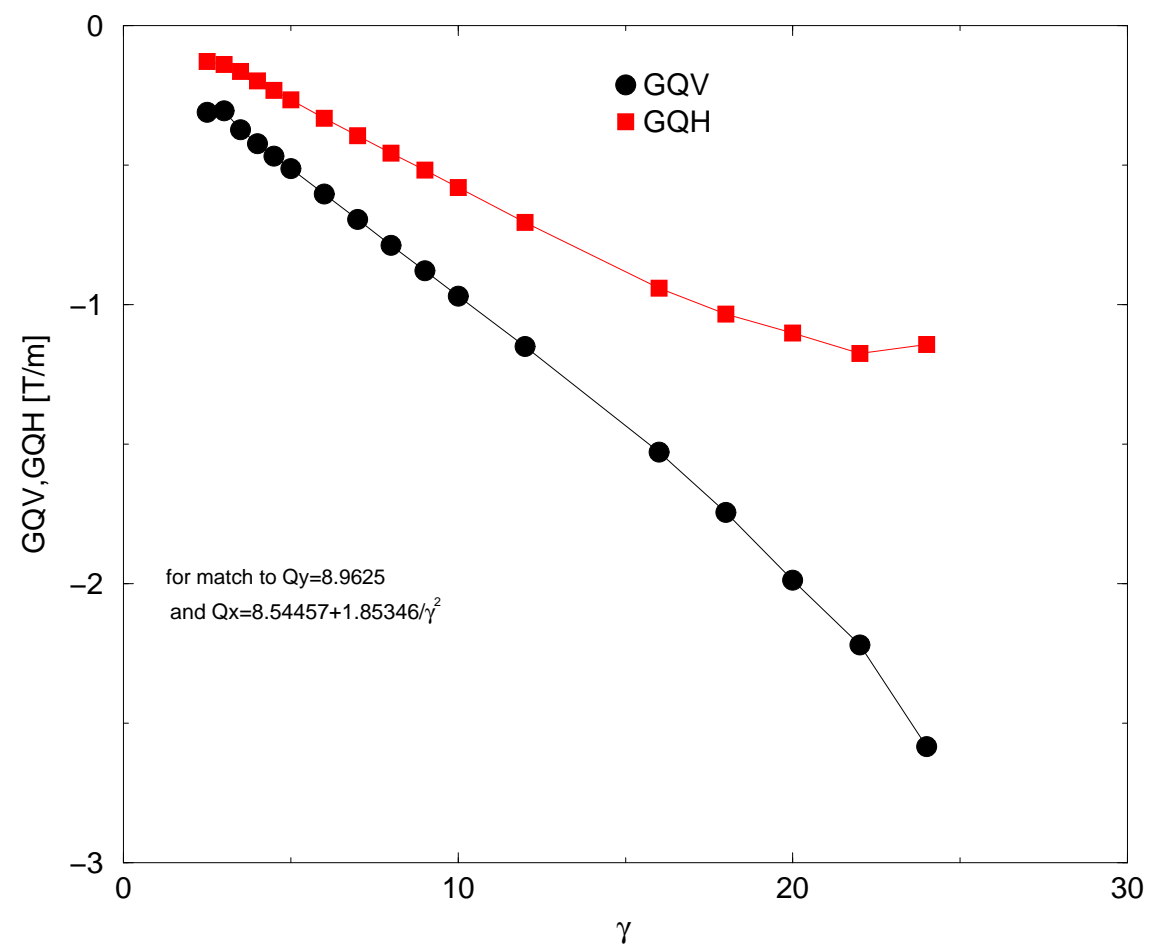

AUL 040414-02

Figure 25: Case 4; gradients in the tune quadrupoles.

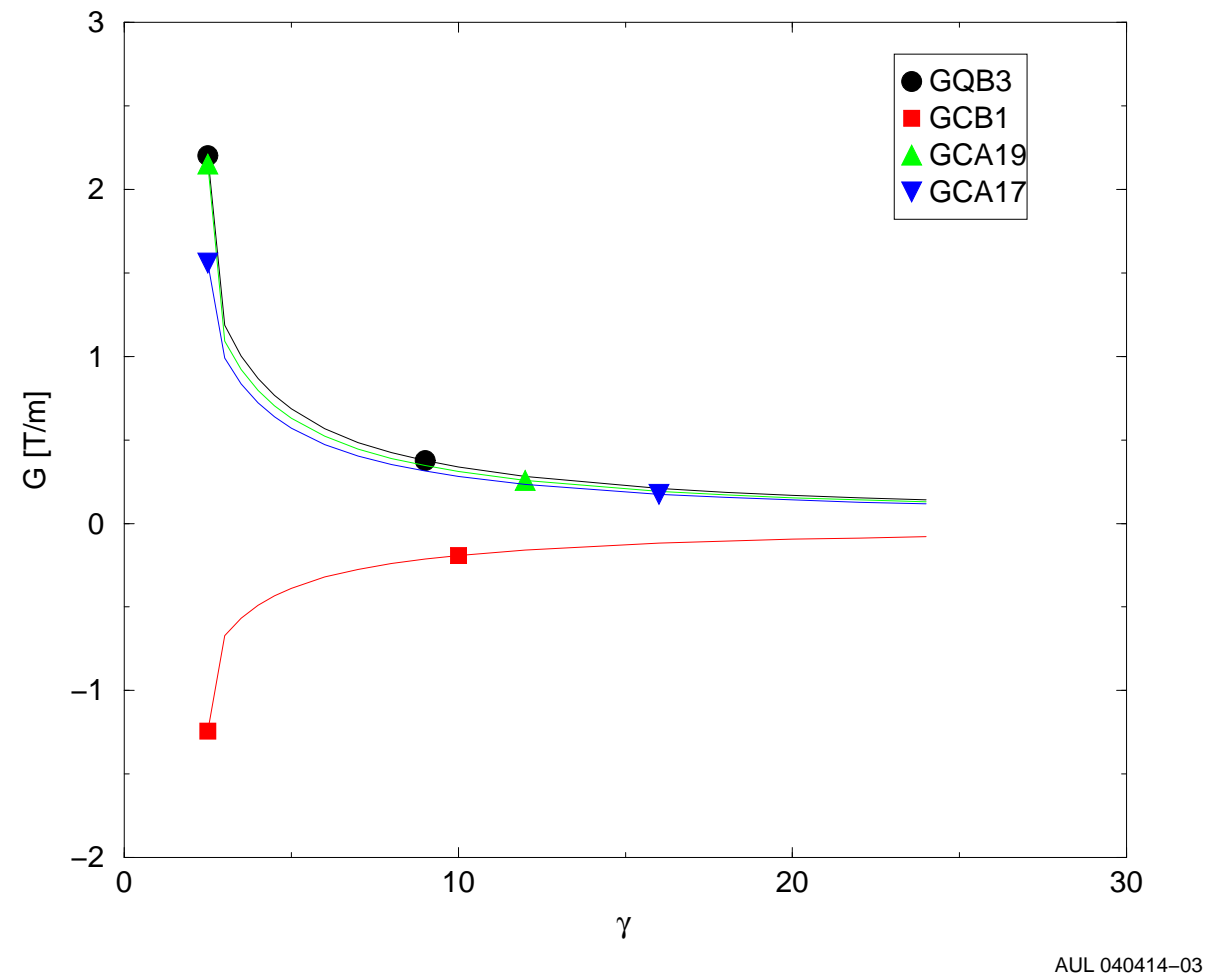

Figure 26: Case 4: gradient of the matching quadrupoles. QCA17 is lumped with QH and QCB3 with $\mathrm{QV}$, respectively. 


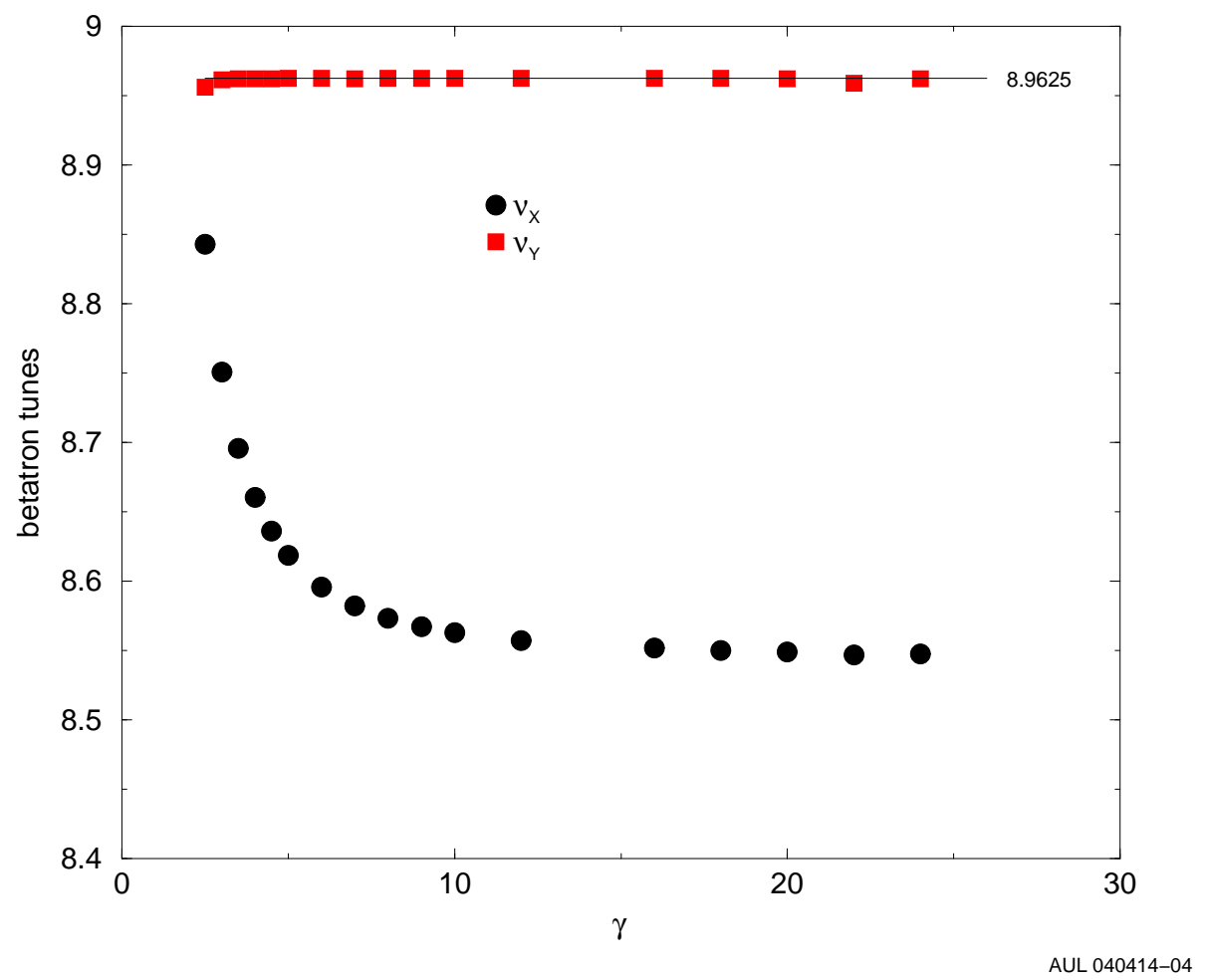

Figure 27: Case 4: betatron tunes after rematch.

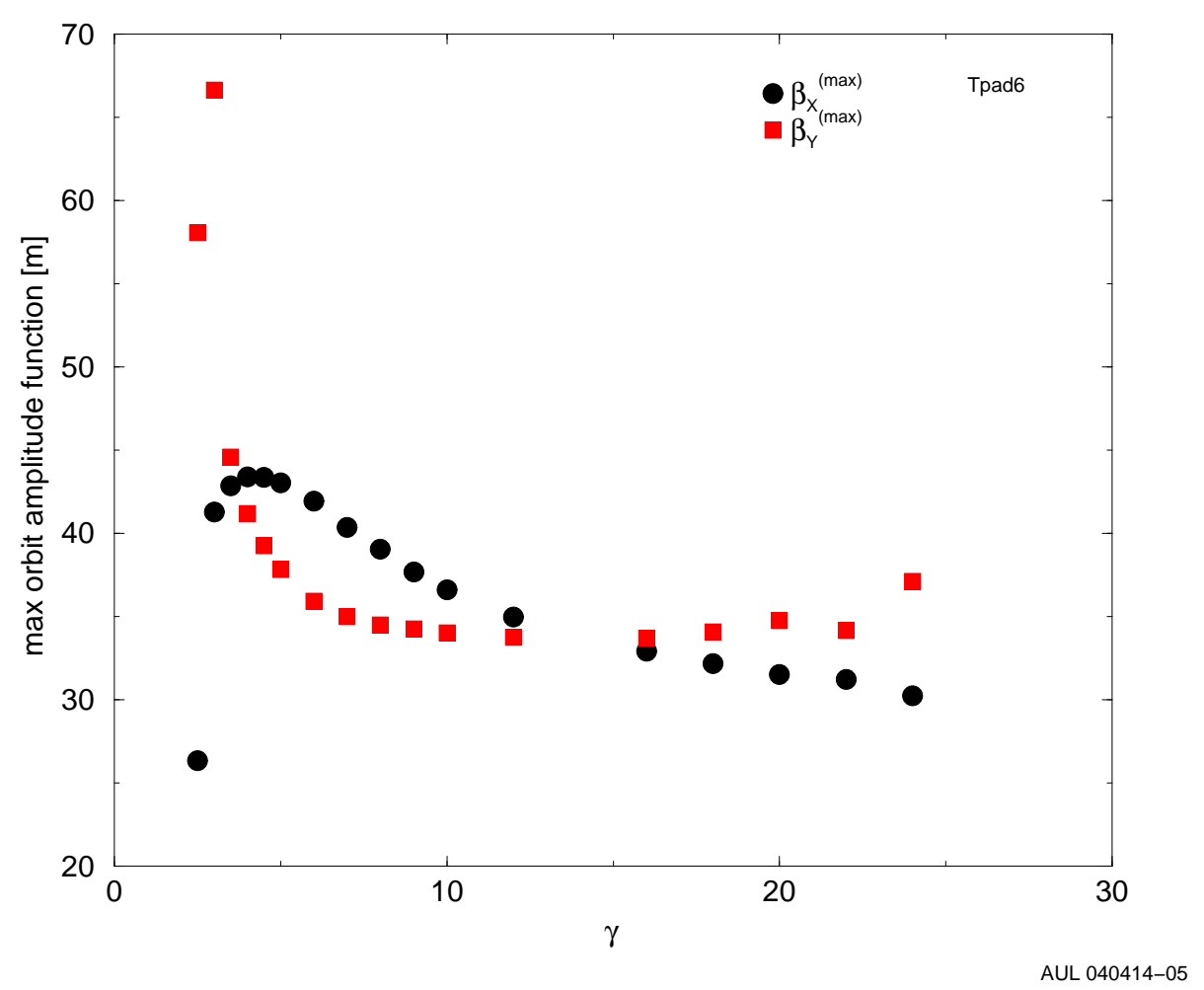

Figure 28: Case 4: maximum beta. 
Table 3: Case 4: Matching quadrupole strengths.

\begin{tabular}{rrrrr}
\hline$\gamma$ & GCB3 & GCB1 & GCA19 & GCA17 \\
\hline 2.5 & 2.2023105 & -1.2429583 & 2.1513402 & 1.5579697 \\
3.0 & 1.1873906 & -0.6714310 & 1.0919365 & 0.9886248 \\
3.5 & 1.0012957 & -0.5662003 & 0.9208017 & 0.8336814 \\
4.0 & 0.8671475 & -0.4903439 & 0.7974377 & 0.7219895 \\
4.5 & 0.765461 & -0.4328436 & 0.7039260 & 0.6373253 \\
5.0 & 0.6855403 & -0.3876509 & 0.6304298 & 0.5707828 \\
6.0 & 0.5676813 & -0.3210054 & 0.5220455 & 0.4726530 \\
7.0 & 0.4847502 & -0.2741105 & 0.4457812 & 0.4036044 \\
8.0 & 0.4231246 & -0.2392633 & 0.3891097 & 0.3522948 \\
9.0 & 0.3754858 & -0.2123251 & 0.3453006 & 0.3126306 \\
10. & 0.3375367 & -0.1908661 & 0.3104022 & 0.2810340 \\
12. & 0.2808475 & -0.1588102 & 0.2582702 & 0.2338344 \\
16. & 0.2103141 & -0.1189258 & 0.1934070 & 0.1751081 \\
18. & 0.1868690 & -0.1056684 & 0.1718466 & 0.1555877 \\
20. & 0.1681326 & -0.0950736 & 0.1546165 & 0.1399877 \\
22. & 0.1528146 & -0.0864117 & 0.1405299 & 0.1272339 \\
24. & 0.1400569 & -0.0791976 & 0.1287978 & 0.1166118
\end{tabular}

Table 4: Case 4: Tune quadrupole and tune after RETUNE.

\begin{tabular}{rrrrrrrrr}
\hline$\gamma$ & $\mathrm{XQV}$ & $\mathrm{XQH}$ & $\mathrm{GQV}$ & $\mathrm{GQH}$ & $\nu_{x}$ & $\nu_{y}$ & $\beta_{x}$ & $\beta_{y}$ \\
\hline 2.5 & 69.50638 & -30.51519 & -0.3109822 & -0.1295304 & 8.842867 & 8.956203 & 26.362331 & 58.077715 \\
3.0 & 68.45034 & -33.05269 & -0.3063162 & -0.1406640 & 8.750745 & 8.961549 & 41.307977 & 66.648328 \\
3.5 & 83.75624 & -38.69407 & -0.3739693 & -0.1654080 & 8.695918 & 8.962372 & 42.886177 & 44.586139 \\
4.0 & 94.99565 & -46.33294 & -0.4236824 & -0.1988951 & 8.660426 & 8.962479 & 43.419881 & 41.204526 \\
4.5 & 105.18205 & -54.25947 & -0.4687613 & -0.2336208 & 8.636105 & 8.962499 & 43.370263 & 39.281710 \\
5.0 & 115.38584 & -62.03082 & -0.5139379 & -0.2676442 & 8.618712 & 8.962502 & 43.063738 & 37.829604 \\
6.0 & 135.96795 & -76.98028 & -0.6051213 & -0.3330307 & 8.596057 & 8.962500 & 41.958611 & 35.948805 \\
7.0 & 156.31732 & -91.35123 & -0.6953402 & -0.3958078 & 8.582420 & 8.962445 & 40.358093 & 35.011962 \\
8.0 & 177.31752 & -105.4806 & -0.7885025 & -0.4574541 & 8.573531 & 8.962500 & 39.077189 & 34.497822 \\
9.0 & 197.89848 & -119.5168 & -0.8798514 & -0.5186190 & 8.567491 & 8.962515 & 37.700742 & 34.273267 \\
10. & 218.35955 & -133.8103 & -0.9707036 & -0.5808307 & 8.563105 & 8.962500 & 36.607276 & 34.031845 \\
12. & 259.07449 & -162.7503 & -1.1515578 & -0.7065663 & 8.557442 & 8.962500 & 34.979993 & 33.775256 \\
16. & 344.25203 & -217.00616 & -1.5299898 & -0.9415787 & 8.551810 & 8.962500 & 32.932564 & 33.716489 \\
18. & 392.83004 & -238.45247 & -1.7457474 & -1.0342752 & 8.550291 & 8.962500 & 32.187046 & 34.095397 \\
20. & 447.58488 & -254.32295 & -1.9888253 & -1.1028244 & 8.549204 & 8.962497 & 31.533243 & 34.785932 \\
22. & 499.99999 & -271.37590 & -2.2213962 & -1.1764553 & 8.546819 & 8.958973 & 31.234811 & 34.189181 \\
24. & 581.88058 & -263.89028 & -2.5845079 & -1.1441361 & 8.547788 & 8.962497 & 30.265418 & 37.119023
\end{tabular}




\section{Conclusions}

Matching of the Warm Snake to the AGS lattice using four "Courant" quadrupoles is possible. For the quadrupoles there is no much space available, therefore they will present a severe challenge to the Engineers. A perfect match, i.e. one that will leave the optics in the part of the lattice outside the section containing the snake is also possible in theory, but it is not desirable because the corresponding currents in the matching quadrupoles do not vary according to smooth curves vs. energy. A reasonable matching is obtain by letting the curves decrease with beam energy following a $(B \rho)^{-2}$ function.

\section{References}

[1] E.D.Courant: Matching Quadrupoles for AGS Helical Snake. In: SPIN2002 15.th International Spin Physics Symposium, Upton, NY, 9-14 Sept. 2002, AIP Conference Proceedings, volume 675, 799-803.

[2] A.U.Luccio: Cold AGS Snake Optimization by Modeling. Technical Report C-A/AP/128, Brookhaven National Laboratory. Upton, NY, Upton, NY, December 2003. 